\title{
Vascular Sema3E-Plexin-D1 Signaling Reactivation Promotes Post-stroke Recovery through VEGF Downregulation in Mice
}

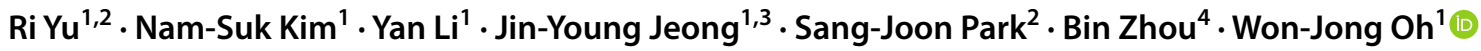

Received: 24 November 2020 / Revised: 21 April 2021 / Accepted: 26 April 2021 / Published online: 12 May 2021

(c) The Author(s) 2021

\begin{abstract}
Post-stroke vascular remodeling, including angiogenesis, facilitates functional recovery. Proper vascular repair is important for efficient post-stroke recovery; however, the underlying mechanisms coordinating the diverse signaling pathways involved in vascular remodeling remain largely unknown. Recently, axon guidance molecules were revealed as key players in injured vessel remodeling. One such molecule, Semaphorin 3E (Sema3E), and its receptor, PlexinD1, control vascular development by regulating vascular endothelial growth factor (VEGF) signaling. In this study, using a mouse model of transient brain infarction, we aimed to investigate whether Sema3E-Plexin-D1 signaling was involved in cerebrovascular remodeling after ischemic injury. We found that ischemic damage rapidly induced Sema3e expression in the neurons of peri-infarct regions, followed by Plexin-D1 upregulation in remodeling vessels. Interestingly, Plexin-D1 reemergence was concurrent with brain vessels entering an active angiogenic process. In line with this, Plxndl ablation worsened neurological deficits, infarct volume, neuronal survival rate, and blood flow recovery. Furthermore, reduced and abnormal vascular morphogenesis was caused by aberrantly increased VEGF signaling. In Plxndl knockout mice, we observed significant extravasation of intravenously administered tracers in the brain parenchyma, junctional protein downregulation, and mislocalization in regenerating vessels. This suggested that the absence of Sema3E-Plexin-D1 signaling is associated with blood-brain barrier (BBB) impairment. Finally, the abnormal behavioral performance, aberrant vascular phenotype, and BBB breakdown defects in Plxndl knockout mice were restored following the inhibition of VEGF signaling during vascular remodeling. These findings demonstrate that Sema3E-Plexin-D1 signaling can promote functional recovery by downregulating VEGF signaling in the injured adult brain.
\end{abstract}

Keywords Plexin-D1 $\cdot$ Axon guidance $\cdot$ Stroke $\cdot$ Vascular recovery $\cdot$ Blood-brain barrier

Won-Jong $\mathrm{Oh}$

ohwj@kbri.re.kr

1 Neurovascular Biology Laboratory, Neurovascular Unit Research Group, Korea Brain Research Institute, Daegu 41062, Republic of Korea

2 College of Veterinary Medicine, Kyungpook National University, Daegu 41566, Republic of Korea

3 Department of Brain and Cognitive Sciences, Daegu Gyeongbuk Institute of Science and Technology, Daegu 42988, Republic of Korea

4 Institute of Biochemistry and Cell Biology, Shanghai Institutes for Biological Sciences, Chinese Academy of Sciences, Shanghai 200031, China

\section{Introduction}

Stroke is the most common cause of death and a leading cause of disability worldwide [1,2]. It is accompanied by structural and functional vascular injuries and remodeling [3]; therefore, enhancing angiogenesis in the damaged tissue to promote recovery of nearby brain cells has been proposed as a therapeutic option [3, 4]. However, despite tremendous efforts to develop pharmacological therapies promoting cerebrovascular repair, there are no definitive remedies. This is mainly due to the varying degrees of severity from earlyonset to late chronic pathology and the multifaceted cellular and molecular events that occur during repair [5-7]. For example, treatment with tissue plasminogen activator is considered the gold standard for ischemic stroke; however, one of its side effects is an enhanced inflammatory response 
in brain capillaries and neuronal cell damage $[8,9]$. The brain vasculature has a unique structure that is composed of endothelial cells, neurons, astrocytes, and pericytes. This is known as the neurovascular unit (NVU), where vessels tightly regulate the passage of substances between the bloodstream and brain parenchyma via the blood-brain barrier (BBB) $[10,11]$. Neural activity and vascular dynamics are tightly coupled in the NVU; the connection and bidirectional communication between the two systems is crucial for proper brain function $[12,13]$. Because of the importance of such integrative interaction, neovascularization and vascular repair in the brain after injury require stepwise processes and regulatory mechanisms to reconstruct a functional NVU [14-16].

Previous studies have shown that angiogenesis rapidly occurs post-ischemia in the brain. This contributes to functional recovery and prolonged survival in human patients and experimental rodent models $[3,16,17]$. Indeed, endothelial cell proliferation occurs at $12-24 \mathrm{~h}$ following a stroke, and active angiogenesis in the peri-infarcted region is observed 3-4 days after an ischemic insult in humans [15]. However, little is known about the molecular mechanism orchestrating vascular remodeling during repair processes in the post-ischemic brain. To coordinate a highly branched and hierarchically organized vascular network, endothelial cells grow and connect according to the multiple steps during angiogenesis [18]. VEGF and its receptor, VEGF receptor 2 (VEGFR2), are expressed by endothelial cells; they are master mediators of vascular network establishment during both developmental and pathological angiogenesis [19, 20]. After cerebral ischemia, both VEGF and VEGFR2 are mainly expressed in the ischemic border, known as the peri-infarct region, where the vessels remain salvageable. Therefore, this is considered a treatable target [21-23]. Interestingly, VEGF signaling is associated with neuroprotection and neurogenesis in ischemic stroke models [24-28]. However, it produces harmful effects, such as BBB breakdown, that are dependent on administration timing and dosage after stroke induction $[29,30]$. Therefore, elucidating the balanced mechanism and precise activation dynamics of VEGF signaling is of high importance [31].

During the angiogenic process, endothelial tip cells use the same guidance molecules as neuronal growth cones to regulate proper vascular network formation $[10,12]$. These cues also play a crucial role during vascular remodeling induced by ischemic damage [32]. One such guidance cue is Semaphorin 3E (Sema3E) and its receptor, Plexin-D1. Sema3E is one of seven class-3 secreted semaphorins. It transduces its signal via direct binding to the Plexin-D1 receptor independently of neuropilins, the primary binding receptor of other class-3 semaphorins $[13,33]$. In the nervous system, the Sema3E-Plexin-D1 axis provides a repulsive signal by inhibiting axonal growth or restricting synapse formation $[34,35]$. However, its mode of action on vascular topology varies depending on the temporal and spatial expression patterns of Sema3E and Plexin-D1 [33]. For example, the absence of Sema3E-Plexin-D1 signaling leads to excessive vessel outgrowth in the developing somite or decreased vessel sprouting in the retina $[13,36]$. Moreover, Sema3E-Plexin-D1 signaling is associated with ischemiainduced angiogenesis in the periphery tissue and retina [13, 37], but its activity in the central nervous system has not been elucidated. A few studies have revealed that Sema3EPlexin-D1 signaling is interwoven with VEGF signaling under normal or pathological conditions [36-38]. In the developing and pathological retinal vasculature, Sema3EPlexin-D1 signaling modulates the angiogenic process at sprouting ends through a VEGF-induced negative feedback mechanism [36, 37]. Because Sema3E-Plexin-D1 signaling is actively involved in many angiogenic conditions, we speculated that this signaling contributes to vascular remodeling after brain damage, such as in ischemic stroke. In this study, we investigated whether Sema3E-Plexin-D1 signaling was necessary for the development of functional brain vasculature during vascular remodeling after an ischemic insult.

\section{Materials and Methods}

\section{Animals}

Plxndl flox/flox [36] and Mfsd2a-CreERT2 mice [39] were maintained on a C57Bl/6 J (\#000664, Jax) background. Rosa26-RFP mice (( Ai9; \#007909, Jax)) were purchased from the Jackson Laboratory and maintained on a C57B1/6 J background. To generate the endothelial cell-specific inducible Plxndl knockout mice, tamoxifen (T5648, Sigma) was dissolved in corn oil and administered to male mice 14 days before surgery. Male mice received 0.1-0.2 mg tamoxifen per gram of body weight by oral gavage. All protocols for animal experiments were approved by the Institutional Animal Care and Use Committee of Korea Brain Research Institute (IACUC-18-00008). All experiments were performed according to the National Institutes of Health Guide for the Care and Use of Laboratory Animals and ARRIVE guidelines.

\section{Ischemic Brain Mouse Model}

The transient middle cerebral artery occlusion (tMCAO) mouse model, whereby a temporary reduction of blood flow followed by reperfusion generates cerebral ischemic injury in the cortex and striatum, was performed as described previously [40]. Briefly, anesthesia was induced with 5\% isoflurane in an anesthetic chamber and maintained with $1.5 \%$ isoflurane via a nose cone. Rectal temperature was 
maintained at $37.0 \pm 0.5^{\circ} \mathrm{C}$ during surgery and the recovery period using a $37{ }^{\circ} \mathrm{C}$ heating pad. A $1-\mathrm{cm}$-long midline skin incision was performed in the neck area. The right common carotid artery (CCA) was carefully dissected free from the surrounding nerves and tied off using a 7-0 silk suture. The right external carotid artery was isolated and cauterized 2-3 $\mathrm{mm}$ distal to the bifurcation, and a vessel clip was placed just before the CCA bifurcation to avoid retrograde flow at the time of arteriotomy. An arteriotomy was performed between the proximal ligated suture and vessel clip on the CCA with micro-scissors. Next, a siliconerubber-coated 6-0 nylon filament (602145PK10, Doccol) was inserted into the CCA and advanced for $9 \mathrm{~mm}$ to the carotid bifurcation along the internal carotid artery (ICA) and origin of the middle cerebral artery (MCA). Next, the neck incision was sutured and the mouse was placed in a $37^{\circ} \mathrm{C}$ nursing box to recover from anesthesia. After $30 \mathrm{~min}$ of occlusion, the filament was removed to restore the blood flow to the MCA. In sham-operated mice, the ICA was surgically prepared for filament insertion, but it was not inserted.

\section{In Situ Hybridization}

In situ hybridization (ISH) was performed under RNase-free conditions by standard procedures [36]. After fixation in 4\% paraformaldehyde (PFA) for $20 \mathrm{~min}, 14-\mu \mathrm{m}$-thick cryosections sections were preincubated in hybridization buffer ( $5 \times$ Denhardt's solution, $5 \times$ saline sodium citrate (SSC), $50 \%$ formamide, $0.25 \mathrm{mg} / \mathrm{ml}$ Baker yeast tRNA, and $0.2 \mathrm{mg} /$ $\mathrm{ml}$ salmon sperm DNA) for at least $1 \mathrm{~h}$ at room temperature. Next, sections were hybridized in the same buffer containing the indicated digoxigenin-conjugated riboprobe at $60{ }^{\circ} \mathrm{C}$ overnight. After hybridization, sections were washed in serial SSC buffer, formamide solution, and preincubated in buffer 1 (100 mM Tris-HCl, pH 7.5, $150 \mathrm{mM} \mathrm{NaCl}$ ) plus $1 \%$ blocking reagent (Roche) for $1 \mathrm{~h}$ at room temperature. Next, sections were incubated with sheep anti-digoxigenin-alkaline phosphatase (AP) antibody (1:3000, Roche) for $90 \mathrm{~min}$ at room temperature, washed in the buffer 1 , and incubated in AP buffer (100 mM Tris- $\mathrm{HCl}, \mathrm{pH} 9.5,100 \mathrm{mM} \mathrm{NaCl}$, $5 \mathrm{mM} \mathrm{MgCl}_{2}$ ) containing 4-nitro blue tetrazolium chloride (NBT, Roche), 5-bromo-4-chloro-3-indolyl-phosphate (BCIP, Roche), and levamisole (1359302, Sigma). Purple precipitates were visualized. After mounting with coverslips, the samples were analyzed via confocal laser-scanning microscopy with a Nikon Eclipse Ti-U microscope or Leica TCS SP8 confocal microscope.

For subsequent immunostaining with anti-ColIV (1:500, ab6586, Abcam) after ISH, sections were washed in $1 \times$ phosphate-buffered saline (PBS) several times and post-fixed in 4\% PFA for 5 min. After fixation, all procedures were performed as described in the immunohistochemistry section. For the double fluorescence ISH, the tyramide signal amplification method with minor modification was used according to the manufacturer's instructions (NEL753001KT, PerkinElmer) [34]. The following antisense riboprobes were used: Plxnd1 [36], Sema3e[13], and Snap25 riboprobes were designed based on the ISH database provided by the Allen Institute for Brain Science.

\section{AP-Ligand Preparation and Binding Analysis}

AP-conjugated Sema3E ligands were generated in HEK293T cells, and ligand binding experiments were performed as described previously [36]. Briefly, the AP-Sema3E expression construct was transfected into cells by LipofectAMINE 2000 (11668019, Invitrogen) and cultured overnight in Dulbecco's Modified Eagle's Medium (DMEM, 11995-065, Gibco) containing $10 \%$ fetal bovine serum (FBS). Next, the medium was replaced with OPTI-MEM (31985-070, Gibco) and harvested at 5 days post-transfection. The collected conditioned medium was filtered to increase ligand concentration.

For AP-Sema3E binding analysis, $20-\mu \mathrm{m}$-thick cryosections were fixed in cold methanol for $8 \mathrm{~min}$ and preincubated in $1 \times \mathrm{PBS}$ containing $4 \mathrm{mM} \mathrm{MgCl}_{2}$ and $10 \% \mathrm{FBS}$ for $1 \mathrm{~h}$. Next, a binding solution $\left(1 \times \mathrm{PBS}-\mathrm{MgCl}_{2}\right.$ plus $20 \mathrm{mM}$ HEPES, pH 7.0) containing $2 \mathrm{nM}$ AP-Sema3E was applied, and sections were incubated for $2 \mathrm{~h}$ at room temperature. After five washes in $1 \times \mathrm{PBS}-\mathrm{MgCl}_{2}$, sections were briefly soaked in acetone-formaldehyde fixative (60\% acetone, $1.1 \%$ formaldehyde, and $20 \mathrm{mM}$ HEPES $\mathrm{pH}$ 7.0) and heat-inactivated in $1 \times \mathrm{PBS}$ at $65^{\circ} \mathrm{C}$ for $2 \mathrm{~h}$. Next, sections were incubated in AP buffer with NBT and BCIP overnight at room temperature. The subsequent immunostaining procedure was performed as described in the immunohistochemistry section. For quantification, three brain sections per animal were analyzed and averaged the mean signal intensity per image.

\section{Neurological Scoring}

A neurological deficit grading system [41] was used to evaluate mice at 1, 2, 3, 4, 5, 6, and 7 days after tMCAO. The scores were as follows: 0 , no neurological deficit; 1 , forelimb flexion when suspended by the tail, or failure to fully extend forepaw; 2 , shoulder adduction when suspended by the tail; 3 , reduced resistance to lateral push; 4 , spontaneous movement in all directions with unilateral circling only if pulled by the tail; 5 , spontaneous unilateral circling; 6 , walk only when stimulated; 7 , no response to stimulation; and 8 , stroke-related death.

\section{Magnetic Resonance Imaging}

Magnetic resonance imaging (MRI) experiments were performed using a Bruker 9.4 preclinical MRI (BioSpec 94/20 
USR; Laboratory Animal Center of Daegu-Gyeongbuk Medical Innovation Foundation, Korea). Briefly, mice were anesthetized with a continuous supply of $1.5 \%$ isoflurane. Respiratory rates were monitored continuously. A fast spin-echo sequence (4000/12.5 ms of repetition time/ echo time) was employed for T2-weighted imaging and a spin-echo sequence (700/7 ms of repetition time/echo time) for T1-weighted imaging. For all scans, the field of view was $20 \times 20 \mathrm{~mm}^{2}$, slice thickness $3.0 \mathrm{~mm}$, number of slices 18 , and matrix size $256 \times 256$, except for the diffusion sequence where the matrix size was $128 \times 128$. Pre-contrast T1-weighted images were acquired before gadoterate meglumine (Gd-DOTA) injection. Post-contrast T1-weighted images were acquired $30 \mathrm{~min}$ after intravenous injection of Gd-DOTA $(0.2 \mathrm{ml} / \mathrm{kg}$ body weight $)$ with normal saline. A region of interest (ROI) was drawn in the peri-infarct and extravasation areas on images using the ImageJ software (National Institutes of Health, Bethesda, MD; http://imagej. nih.gov/ij/).

\section{Immunohistochemistry}

For immunostaining with floating samples, brains were fixed in 4\% PFA overnight and equilibrated with $30 \%$ sucrose in $1 \times$ PBS. Mouse brain sections were cut into $30-\mu \mathrm{m}$ slices on a cryostat (Leica Microsystems Inc., Buffalo Grove, IL, USA). Next, they were permeabilized in PBST (PBS containing $0.3 \%$ Triton X-100) for 10 min, blocked with $1 \%$ bovine serum albumin (BSA) in PBST for $60 \mathrm{~min}$ at room temperature, and incubated in primary antibody diluted in blocking solution overnight at $4{ }^{\circ} \mathrm{C}$. The following primary antibodies were used: anti-CD31 (1:500, 553370, BD Bioscience), anti-Collagen IV (1:500, ab6586, Abcam), anti-NeuN (1:1000, MAB377, Merck), anti-Ter-119 (1:100, MAB1125, R\&D System), and anti-MAP-2 (1:500, ab32454, Abcam). After washing with PBST three times, sections were incubated for $1 \mathrm{~h}$ with Alexa Fluor 488-, 594-, or 647-conjugated secondary antibodies (1:1000, Invitrogen). For negative controls, brain sections were stained with secondary antibodies alone.

For BBB junctional protein immunostaining, brains were isolated, immediately snap-frozen with liquid nitrogen, and cut (14- $\mu \mathrm{m}$-thick sections) on a cryostat. Sections were fixed in cold $100 \%$ acetone for 15 min followed by cold $100 \%$ methanol for $20 \mathrm{~min}$. Permeabilization, blocking, and antibody incubation were performed as described above. The following primary antibodies were used: anti-ZO-1 (1:500, 61-7300, ThermoFisher), anti-claudin5 (1:200, 35-2500, Invitrogen), anti-claudin5-488 ( 1:500, 352588, Invitrogen), and anti-Occludin (1:200, 33-1500, Invitrogen). Images were collected under a Nikon Eclipse Ti-U microscope (Nikon, Japan) or Leica TCS SP8 confocal microscope (Leica, Germany). Image processing was performed using the ImageJ, Adobe Photoshop (Adobe Photoshop CC2019), and Angio Tool (National Cancer Institute, Gaithersburg, MD, USA) software.

\section{Measurements of Infarct Volume}

Infarct area was measured by cresyl violet and microtubule-associated protein 2 (MAP2) staining. Mouse brain sections were cut into $30-\mu \mathrm{m}$ slices on a cryostat (Leica Microsystems Inc., Buffalo Grove, IL, USA) from five levels (bregma $-1.6 \mathrm{~mm},-0.8 \mathrm{~mm}, 0 \mathrm{~mm}, 0.8 \mathrm{~mm}$, and $1.6 \mathrm{~mm}$ ) and stained with $1 \%$ cresyl violet solution. In addition, the neuron-specific marker, MAP2, was visualized with antiMAP2 (1:500, ab32454, Abcam) immunofluorescence staining. Sections were digitized and the border between infarct and non-infarct tissue outlined using ImageJ. The infarct volume was calculated by subtracting the volume of the nonlesioned area in the ipsilateral hemisphere from the volume of the whole area in the contralateral hemisphere.

\section{Vascular Labeling and Analysis of Vascular Density}

Animals were intravenously perfused 5 min before euthanasia with Dylight 594-conjugated Lycopersicon esculentum (Tomato) lectin (DL-1177, Vector Laboratories) at a dose of $1 \mathrm{mg} / \mathrm{kg}$ body weight. After perfusion, the brains were dissected, fixed, and then cut into $100-\mu \mathrm{m}$-thick free-floating sections using a vibratome. Vascular area (\%), vessel length $\left(\mu \mathrm{m} / \mu \mathrm{m}^{2}\right)$, and branch point number were calculated using Angio Tool.

For microaneurysm-like capillary vessel analysis, images immunostained with anti-Col IV and anti-Ter-119 antibodies without PBS perfusion procedure were analyzed using ImageJ. The capillaries holding the single lining of red blood cells were defined as a normal capillary. The mean diameter of the capillaries was calculated to determine the cutoff value. Vessels thicker than the cutoff size were considered abnormal. The number and coverage of abnormal $v s$ normal vessels were analyzed per image.

\section{Terminal Deoxynucleotidyl Transferase-Mediated dUTP Nick End-Labeling Staining}

Terminal deoxynucleotidyl transferase dUTP nick end labeling (TUNEL) assay was employed to observe the apoptotic cells. Using the In Situ Cell Death Detection Kit, TMR red (12156792910, Roche), the brain sections were treated according to the manufacturer's protocol.

\section{Immunoblotting}

Brain tissue was collected in a RIPA buffer with a protease inhibitor cocktail (11697498001, Roche) and protein amounts were quantified by a BCA protein assay kit 
(23227, Thermo Fisher Scientific). A total of $40 \mathrm{~g}$ of protein was loaded per well and separated on a sodium dodecyl sulfate (SDS) polyacrylamide gel and transferred to a polyvinylidene fluoride (PVDF) membrane (IPVH00010, Merck) at $100 \mathrm{~V}$ for $90 \mathrm{~min}$. All membranes were blocked with Everyblot blocking buffer (12010020, Bio-rad) for $1 \mathrm{~h}$ and probed overnight with the indicated primary antibodies in blocking buffer at $4{ }^{\circ} \mathrm{C}$. The primary antibodies were rabbit monoclonal anti-Phospho-VEGF Receptor2 (Tyr951) (15D2) antibody (1:1000, 4991, Cell Signaling), rabbit monoclonal anti-Phospho-VEGF Receptor 2 (Tyr1175) (D5B 11) antibody (1:1000, 3770, Cell Signaling), rabbit monoclonal anti-VEGF Receptor 2 (55B11) antibody (1:1000, 2479, Cell Signaling), goat polyclonal anti-Dl14 antibody (1:1000, AF1389, R\&D systems), rabbit monoclonal anti-b-actin antibody (1:5000, 5125, Cell Signaling), rabbit polyclonal anti-ZO-1 antibody (1:1000, 61-7900, Invitrogen), rabbit polyclonal anti-Claudin- 5 antibody ( $1: 1000$, 34-1600, Invitrogen), and rabbit polyclonal anti-Occludin antibody (1:1000, 71-1500, Invitrogen). The membranes were incubated in PBST and appropriate horseradish peroxidase (HRP)-conjugated secondary antibodies. Bands were developed with enhanced chemiluminescence using the Fusion FX7 (Vilber, Germany) and analyzed using Image J.

\section{Whole-Brain Immunostaining and Clearing}

For adult mouse brain clearing, the CUBIC method was used [42] according to the manufacturer's instructions (T3740 and T3741, Tokyo Chemical Industry). After fixation in cold 4\% PFA in $0.1 \mathrm{M}$ phosphate buffer ( $\mathrm{pH} 7.4$ ), the areas of interest in the brain were sectioned in a parallel direction $(3 \mathrm{~mm})$ and washed with $1 \times$ PBS three times for at least $2 \mathrm{~h}$ each time with shaking at room temperature (RT). The samples were then cleared with 1/2-CUBIC-L under shaking at $60 \mathrm{rpm}$ and $37{ }^{\circ} \mathrm{C}$ for $5 \mathrm{~h}$. CUBIC-L was applied for clearing under the same conditions for $48 \mathrm{~h}$. The samples were washed with $1 \times$ PBS containing $0.3 \%$ Triton X-100 (0.3\% PBST) with soft shaking at RT three times for at least $2 \mathrm{~h}$ each time, once overnight and again for $2 \mathrm{~h}$. Next, samples were blocked with 5\% BSA with shaking at $60 \mathrm{rpm}$ at $37{ }^{\circ} \mathrm{C}$ for $3 \mathrm{~h}$, followed by incubation with CD31 antibodies (diluted in 5\% BSA) with shaking at $60 \mathrm{rpm}$ at $37{ }^{\circ} \mathrm{C}$ for $24 \mathrm{~h}$, and washing three times with PBST and shaking at RT for at least $1 \mathrm{~h}$ each time. Corresponding secondary antibodies (diluted in 5\% BSA) were applied to the sample under the same conditions as primary antibody incubations. Next, the samples were rewashed with PBST three times for at least $1 \mathrm{~h}$. Finally, 1/2-CUBIC-R was applied to clear samples with shaking at $60 \mathrm{rpm}$ at $37{ }^{\circ} \mathrm{C}$ for $24 \mathrm{~h}$. CUBIC-R was used for further clearing; it was replaced every 2 days until satisfactory optical transparency was achieved. Cleared samples were stored in CUBIC-R at $4{ }^{\circ} \mathrm{C}$ until imaging. Light-sheet microscopy images were acquired with the LightsheetZ.1 and $5 \times$ EC plan objective lens (Carl Zeiss, Germany).

\section{Cortical Cerebral Blood Flow Measurement}

Real-time two-dimensional cerebral blood flow (CBF) was monitored using a laser speckle contrast imager (PeriCam PSI HR System, Perimed, Sweden). After anesthesia induction, mice were placed in the prone position and the skull was exposed through a cut in the skin at the parietal midline. A camera was placed above the head at a working distance of $10 \mathrm{~cm}$ from the skull surface. This was illuminated with a laser diode $(785 \mathrm{~nm})$ to allow laser penetration through the brain. CBF was recorded before the induction of cerebral ischemia, during cerebral ischemia, and for the first $10 \mathrm{~min}$ of reperfusion. To evaluate CBF changes, the ROI included the cortical area supplied by the MCA.

\section{BBB Permeability}

To assess BBB permeability, Texas red dextran (MW $=10 \mathrm{kDa}$, Invitrogen; $0.1 \mathrm{ml}$ of $10 \mathrm{mg} / \mathrm{ml}$ ) was injected retro-orbitally on 1, 3, and 7 days post-tMCAO. After a 2-h circulation period, mice were perfused transcardially with PBS, followed by ice-cold 4\% PFA in $0.1 \mathrm{M}$ phosphate buffer, and brains were dissected. Brain samples were postfixed in 4\% PFA for 16-18 $\mathrm{h}$ and equilibrated with $30 \%$ sucrose in $1 \times \mathrm{PBS}$. Mouse brain sections that encompassed the MCA region were cut into $30-\mu \mathrm{m}$ slices. Immunofluorescence was performed with antibodies for anti-CD31 (1:500, 553370, BD Bioscience). Alexa594-conjugated mouse IgG (1:500) was used to visualize IgG leakage in the brain. Sections were imaged with a Leica TCS SP8 confocal microscope (Leica, Germany). IgG leakage was quantified with the Fiji software. Brain slices were uniformly thresholded to quantify the total $\operatorname{IgG}$ area. Areas that exceeded threshold levels were defined as leakage areas. The histological procedure was performed as described in the immunohistochemistry section above.

\section{VEGF-R2 Inhibition}

According to the manufacturer's recommendations, mice received daily intraperitoneal injections $(50 \mathrm{mg} / \mathrm{kg})$ of VEGF-R2 inhibitor SU5416 (S8442, Sigma) dissolved in dimethylformamide (DMF). Control animals received intraperitoneal injections of DMF alone.

\section{Statistical Analysis}

Statistical analyses were performed using Prism 8 (GraphPad Software). All results are presented as mean \pm standard error of mean (SEM). At least three mice pairs per experiment 
were used for all histological analyses. For image data quantification, at least three brain sections per mouse were collected and analyzed. All data analyses were performed by a blinded investigator. Statistical significance was determined using two-tailed Mann-Whitney $U$ tests between two groups and one- or two-way ANOVA with Tukey's multiple comparisons test for three or more groups. For ANOVA analysis, the normal distribution of data was using the Shapiro-Wilk normality and Kolmogorov-Smirnov tests. Differences were considered as statistically significant when $p<0.05$.

\section{Results}

\section{Sema3E Is Rapidly Expressed in Peri-infarct Neurons, whereas Plexin-D1 Expression Slowly Increases in Blood Vessels}

We investigated whether Sema3E and Plexin-D1 expression are induced in ischemic tissue after tMCAo. We found a significant and rapid elevation of Sema3e mRNA in the peri-infarct region as early as $6 \mathrm{~h}$ post-tMCAo. In contrast, Plxndl mRNA expression remained relatively unchanged until $24 \mathrm{~h}$ after ischemic injury (Fig. 1a, b). In addition, increased colocalization of Sema3e and the neuronal marker, snap25, indicated that Sema3e expression in peri-infarct neurons is highly responsive to ischemic injury (Fig. 1c).

High Plexin- D1 expression is found in the brain vasculature during development [43]; however, changes in its expression throughout life are not known. To examine Plexin-D1 expression in the brain vasculature, we performed an AP-Sema3E binding assay, which detects PlexinD1 localization through specific ligand-receptor binding capacity $[13,44]$. Consistent with the previous observations, Plexin-D1 was highly expressed throughout the brain vasculature. Interestingly, its expression was dramatically downregulated after birth and barely detectable in adult brain vessels, with the exception of trace amounts in a few larger vessels (Supplemental Fig. 1). Despite no change in Plxndl mRNA expression during the initial phase after ischemic insult, we wondered whether Plexin-D1 could reappear in newly sprouting vessels under ischemic conditions. Since hypoxia induces VEGF and subsequent signaling via VEGFR2 upregulates Plxndl expression in capillary vessels [37], we surmised that the activation of Plxndl expression in remodeling vessels might take time to develop. To test this hypothesis, we analyzed the temporal features of PlexinD1 expression after tMCAo (Fig. 1d, e). Three days after tMCAo, Plexin-D1 protein was expressed in blood vessels near the infarct core (Fig. 1d, area 2), which reached periinfarct vessels by day 7 (Fig. 1d, area 3, and Supplemental Fig. 2). Seven days post-ischemia, most ipsilateral vessels strongly and broadly expressed Plexin-D1 (Fig. 1d, areas 3 and 4) at levels similar to those found in the early developing vasculature (Supplemental Fig. 1). This indicated that vessels were actively being reconstructed. In the contralateral (intact) hemisphere, Plexin-D1 was detected in axonal tracts (Fig. 1d, area 1) and not blood vessels.

\section{Plxnd1 Conditional Ablation in Brain Endothelial Cells Interfered with Recovery after Ischemic Stroke}

Next, to address whether Plexin-D1 was critical for vascular remodeling, we generated an endothelial brain cell-specific Plxndl knockout mouse model (Plxndl ${ }^{i E C K O}$ ). Endothelial cell-specific Plexin-D1 deletion leads to significant perinatal lethality [45]; therefore, it was necessary to ablate Plxnd1 in a temporally controlled manner. To generate this timespecific Plxndl KO mouse, we crossed Plxndl $I^{f l o x f f l o x}$ mice with $M f s d 2 a-C r e E R T$ mice, which express the tamoxifeninducible Cre predominantly in brain endothelial cells and not peripheral vessels [39]. We observed that a single tamoxifen injection into the $M f s d 2 a-C r e E R T ; R O S A 26-R F P$ mouse sufficiently induced reporter gene expression in most adult brain endothelial cells (Supplemental Fig. 3a). Additionally, we confirmed the elimination of Plexin-D1 expression following tamoxifen injection in brain endothelial cells under hypoxic conditions using AP-Sema3E binding analysis (Supplemental Fig. 3b).

We examined the extent of brain damage and vascular alterations after tMCAo in Plxnd $1^{i E C K O}$ mice following the experimental scheme outlined in Fig. 2a. Heterozygous Mfsd2a-CreERT;Plxndl $1^{\text {flox/+ }}$ littermates were used as controls for all experiments because no difference was found in blood flow, vascular structure, survival rate, or infarction size after tMCAo, just as in Plxnd $1^{f l o x / f l o x}$ mice (Supplemental Fig. 4). In addition, we decided to perform all analyses within 7 days after stroke due to ethical considerations. First, Plxnd $I^{i E C K O}$ mice showed a significant decrease in survival rate and body weight in contrast to control littermates (Fig. 2b, c). To examine behavioral performance, we used an 8 -scale neurological deficit grading system [41] and found that Plxnd $I^{i E C K O}$ mice consistently displayed a higher score than the control group for a week (Fig. 2d). On the first day post-stroke, the mean score for control mice was 4 , which improved to 1-3 by post-stroke day 7 . However, the mean score for Plxnd ${ }^{i E C K O}$ mice was 6 on day 1 after tMCAo. A relatively high neuronal deficit phenotype, i.e., spontaneous unilateral circling (scores of 4-5), was maintained a week after tMCAo.

To analyze the histological effects of tMCAo, we measured ischemia-induced lesion volume by $\mathrm{T} 2$-weighted MRI. We observed significantly increased high-intensity regions representing lesions in $P l x n d I^{i E C K O}$ mice on day 7 after tMCAo (Fig. 2e). In addition, the Plxndl $l^{i E C K O}$ mice showed broader infarction areas from the cortex to the 

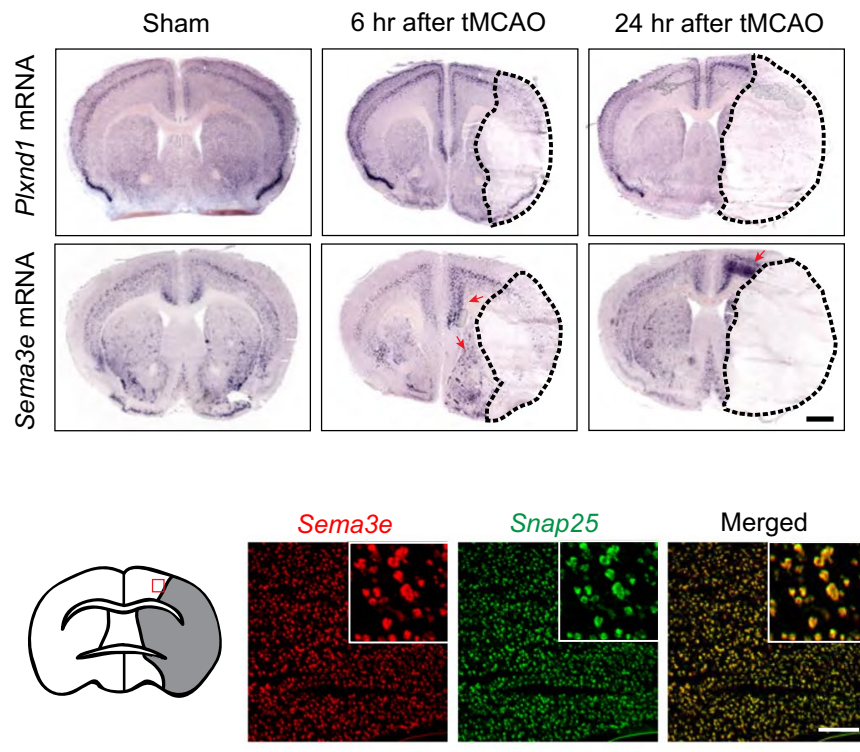

d
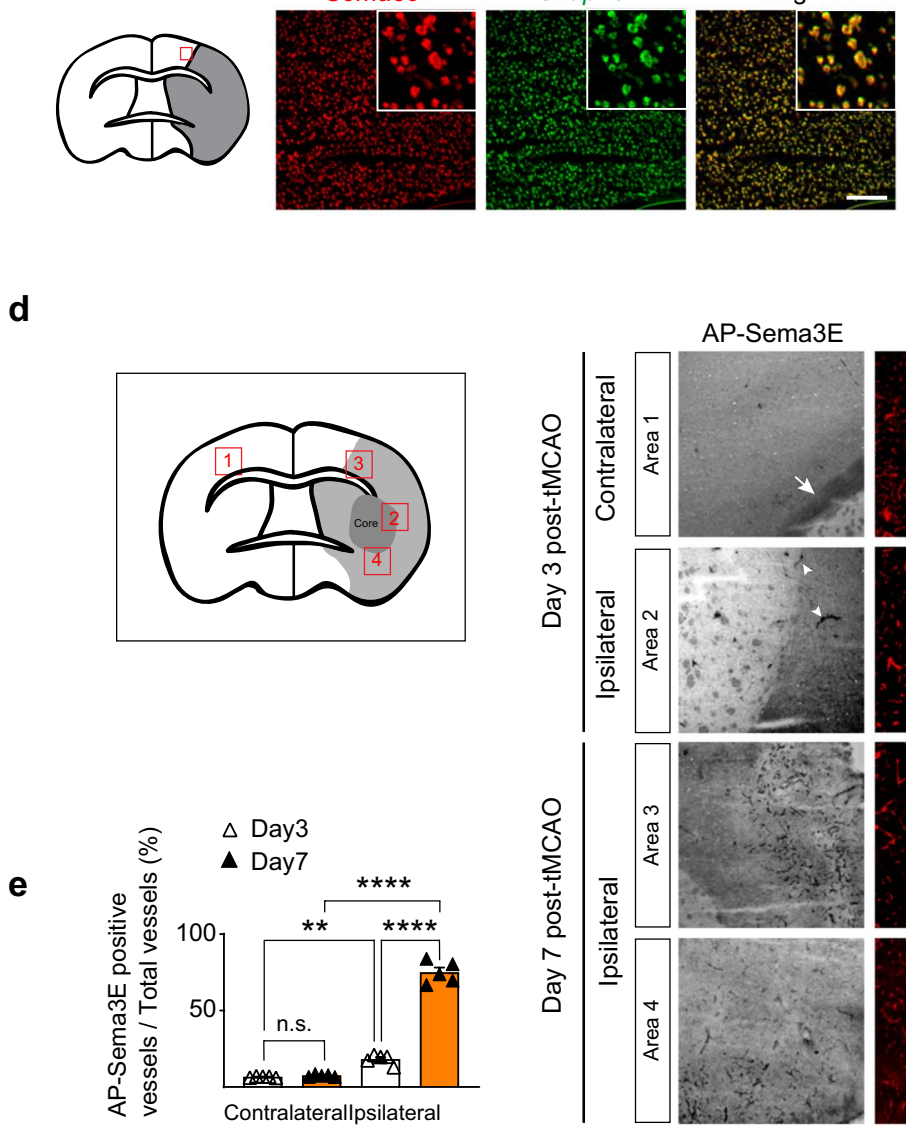

b
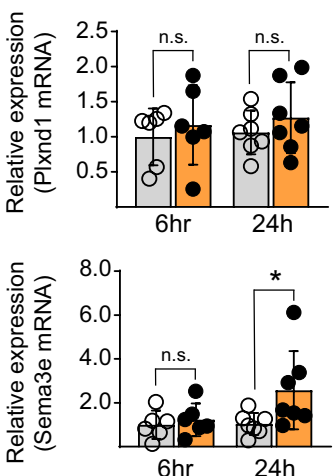

O Contralateral

- Ipsilateral

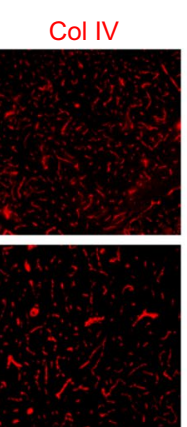

Merged
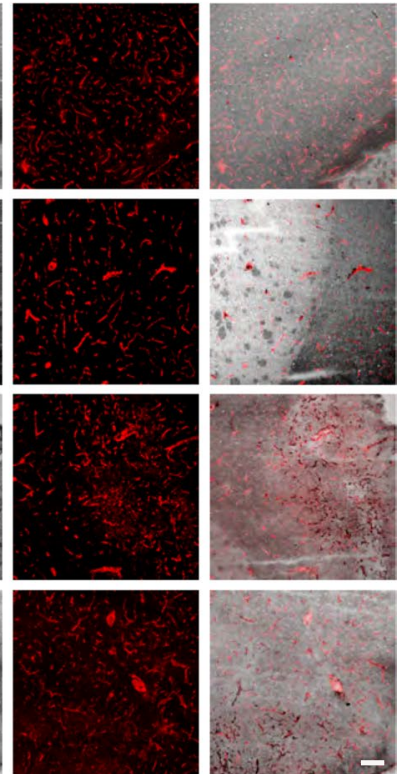

Fig. 1 Sema3e is rapidly expressed in neurons in the peri-infarct region, whereas Plxndl is induced in the remodeling vasculature a In situ hybridization of Sema3e and Plxndl at 6 and $24 \mathrm{~h}$ after tMCAo. The expression of Sema3e mRNA (red arrows), but not Plxnd1 mRNA, was increased in the peri-infarct region. Dotted lines mark regions damaged by tMCAo. Scale bar $=1 \mathrm{~mm}$. b Realtime qPCR for quantitative analysis of mRNA expression in the peri-infarct tissue at 6 and $24 \mathrm{~h}$ after tMCAo $(n=6$ mice $/ 6 \mathrm{~h}$ group, $n=7$ mice $/ 24 \mathrm{~h}$ group). Data are shown as mean \pm SEM. $* p<0.05$; two-tailed Student's $t$-test. c Double fluorescence ISH shows colocalization of Sema3e (red) and snap25 (green) mRNA in the tissue adjacent to the ischemic region at $24 \mathrm{~h}$ after tMCAo. The red box indicates the analyzed brain area, insets are high-resolution images. Scale bar $=100 \mu \mathrm{m}$. d AP-Sema3E binding assay to detect PlexinD1 expression in brain sections (black, left panels) on days 3 and 7 post-tMCAo, and vessels visualized by subsequent collagen IV

(Col IV) immunostaining to detect blood vessels (red, middle panels). Three days after ischemic damage, Plexin-D1-positive vessels (white arrowheads) began to appear on the ipsilateral side, whereas no Plexin-D1 expression was observed on vessels other than those of the neuronal tracts (white arrow) on the contralateral side. Seven days post-tMCAo, most vessels around the damaged region expressed Plexin-D1 (bottom two rows). Numbers in the red boxes indicate image order from top to bottom: 1 , contralateral cortex; 2 , near core damaged region; 3 , peri-infarct region in the ipsilateral cortex; 4 , near damaged core in the ipsilateral area. The light-colored area lacking vessels followed by AP-Sema3E binding was defined as the ischemic core (see also Supplemental Fig. 2). Scale bar $=100 \mu \mathrm{m}$. e Quantification from (d). (three brain sections per mouse, $n=5$ mice/group). Data are shown as mean \pm SEM. $* * p<0.01, * * * * p<0.0001$; one-way ANOVA with Tukey's multiple comparisons 
a

\begin{tabular}{|c|c|c|c|c|}
\hline $\begin{array}{l}8 \sim 10 \text { wk old } \\
\text { male mice }\end{array}$ & Day 0 & Day 1 & Day 3 Day 4 & Day 7 \\
\hline $\begin{array}{c}\uparrow-- \\
\begin{array}{c}\text { Tamoxifen } \\
\text { injection }\end{array}\end{array}$ & $\begin{array}{c}\uparrow \\
\text { IMCAO }\end{array}$ & & $\prod_{\text {Tissue collection }}^{T}$ & MRI, Tissue collection \\
\hline
\end{tabular}

b

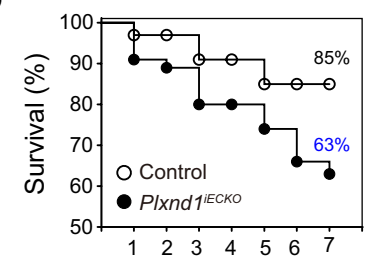

e

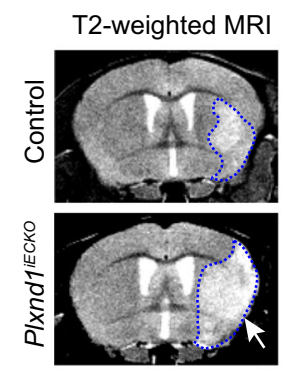

h

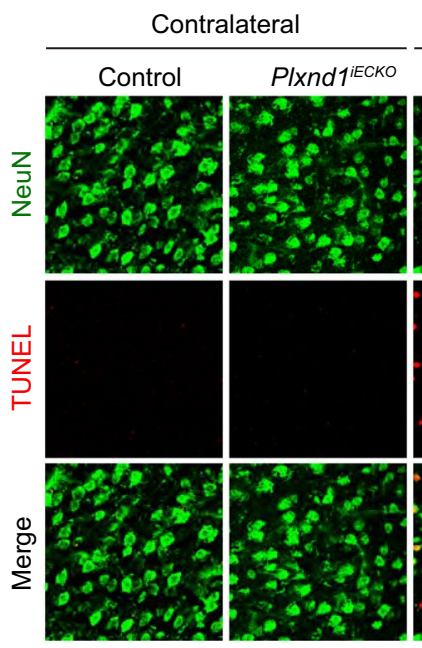

C

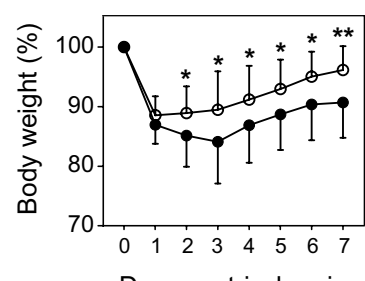

d

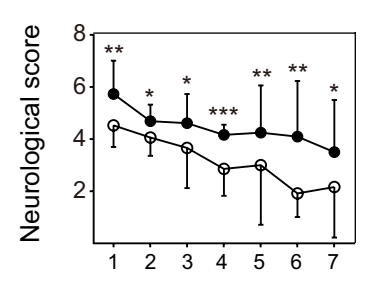

$\mathbf{f}$

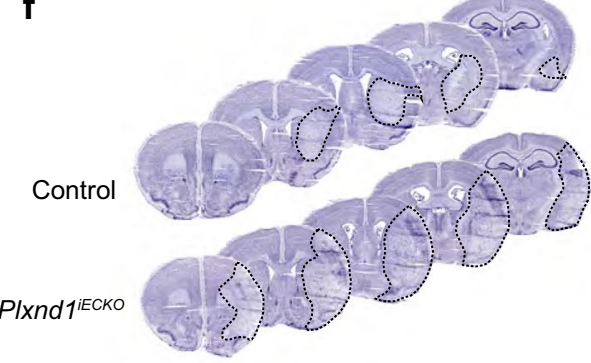

9

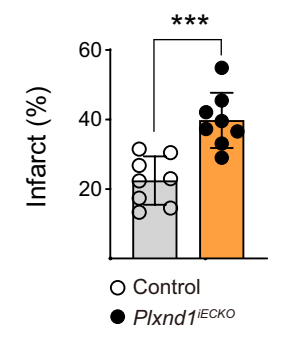

Fig. 2 Brain endothelial cell-specific Plxnd1 knockout leads to severe brain damage in ischemic stroke mice a Schematic illustration of the experimental strategy. b Survival rate changes for 7 days posttMCAo in control (Cre positive Plxnd $1^{\text {flox/+ }}$ ) and Plxnd $1^{\text {iECKO mice }}$ are indicated as open and closed circles, respectively $(\mathrm{n}=34$ mice/ group). c Body weight changes ( $n=20$ mice/group). d Neurological score changes. Detailed score scales are described in the "Materials and Methods" section ( $n=20$ mice/group). e Representative image of T2-weighted MRI coronal sections in control and PlxndI $I^{i E C K O}$ mice. The dotted line in blue indicates the damaged area. White arrows indicate the bigger damaged brain areas in Plxndl ${ }^{i E C K O}$ mice. f Representative image galleries showing Nissl-stained brain sections from control and Plxnd $1^{i E C K O}$ mice at post-tMCAo day 7. The dotted lines in black indicate the infarction region. $g$ Quantification of infarction area analysis in (f) ( $n=8 \mathrm{mice} /$ group). $\mathbf{h}$ Representative images of dead neurons in the peri-infarct region at post-tMCAo day $7 \mathrm{immu}-$ nostained by NeuN (green) and TUNEL (red). In the ipsilateral area, the Plxndl $1^{i E C K O}$ mice show more TUNEL-positive cells compared to the controls. No dead cells were observed in the contralateral side. i Quantification from (h) $(n=5$ mice/control group, $n=6$ mice/Plxnd $l^{\text {iECKO }}$ group). Data are shown as mean \pm SEM. $* p<0.05$, $*^{*} p<0.01, * * * p<0.001$; two-tailed Student's $t$-test (graphs $\mathbf{b}, \mathbf{c}, \mathbf{d}$, and $\mathbf{g}$ ), one-way ANOVA with Tukey's multiple comparison (graph i) 

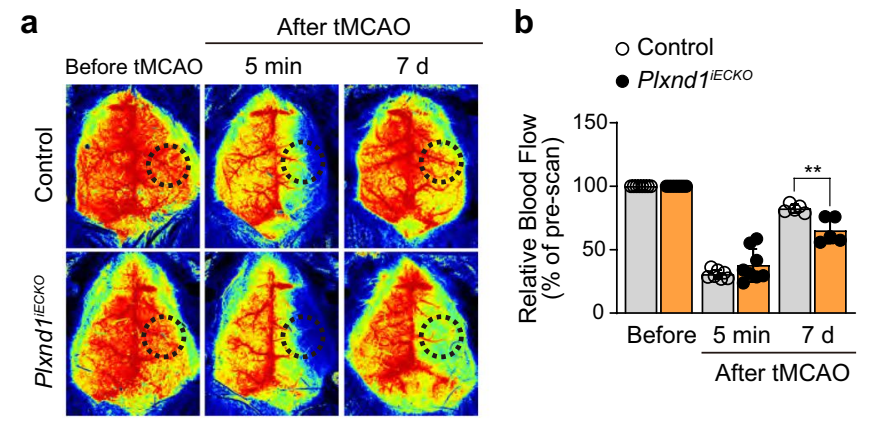

d

Sham
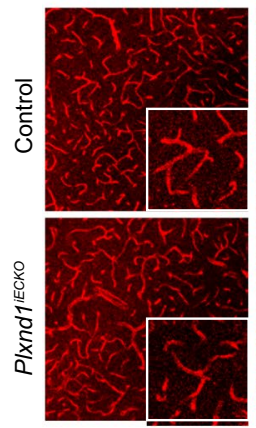

e

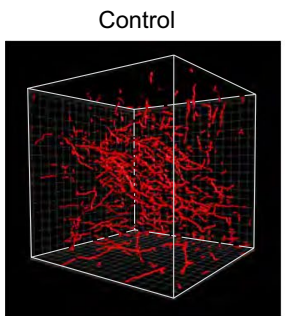

Day 1

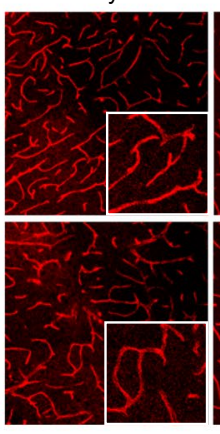

Day 3

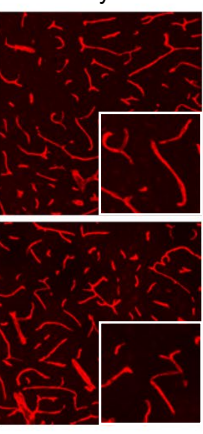

Day 7

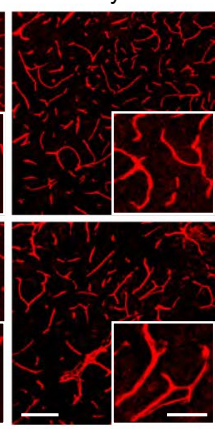

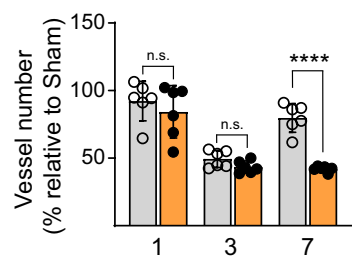
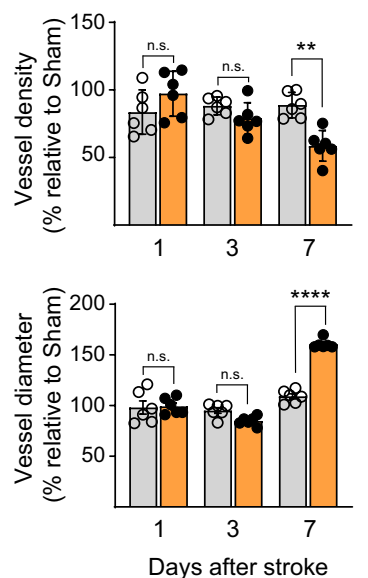

f

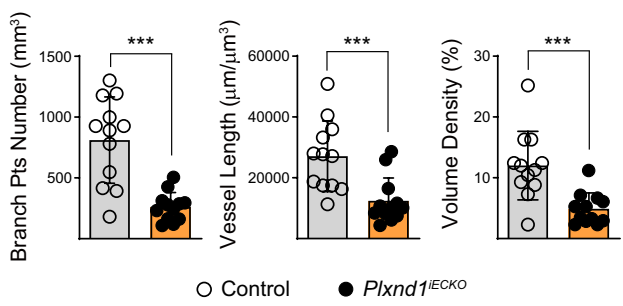

Fig. 3 Plxnd 1 ablation inhibits blood flow recovery and proper vascular network formation a Representative laser speckle images of cerebral blood perfusion in control (Cre positive Plxnd1 $1^{f l o x /+}$ ) and Plxndl ${ }^{i E C K O}$ mouse brains. All images show areas of high and low blood perfusion as yellow-red and blue-black, respectively. The three images represent pre-tMCAo (baseline), post-tMCAo (occlusion), and post-tMCAo day 7 (after reperfusion). b CBF in the cerebral cortical area in (a) was calculated from the circular ROI (black dotted line) and quantified ( $n=5$ mice/group). $\mathbf{c}$ Representative images of temporal changes of microvessel structure in the peri-infarct region of cortex post-tMCAo from control (top panels) and Plxndl $1^{i E C K O}$ (bottom panels) mice. Inset images of each panel showed high-resolution images. Scale bars $=100 \mu \mathrm{m}$ and $50 \mu \mathrm{m}$ (in inset images). d Quantification of the microvascular number, density, and diameter

striatum (Fig. 2f, g). Next, to assess the number of neurons damaged by the ischemic insult, we performed NeuN and TUNEL co-staining and found increased neuronal death in the ipsilateral, damaged hemisphere of Plxndl ${ }^{i E C K O}$ mice when compared with control (heterozygous $M f_{s} d 2 a$ CreERT;Plxndl $I^{f l o x /+}$ ) mice. Damaged neurons were barely detectable in the contralateral side of both the control and Plxndl knockout mice (Fig. 2h, i). At post-tMCAo day for each group ( $n=6$ mice/group). Open and closed circles in black indicate control and PlxndI IECKO mice, respectively. All parameters were analyzed as a relative percentage to sham control data. e Representative $3 \mathrm{D}$ vessel reconstruction analysis images after CD31 immunostaining and tissue clearing. The whole-brain vascular network of the peri-infarct region on day 7 post-tMCAo is analyzed from control and Plxndl $I^{i E C K O}$ mouse brains in $42 \times 10^{6} \mu \mathrm{m}^{3}$ volume. Scale bars $=100 \mu \mathrm{m}$. f Quantification of branch point number, vessel length, and volume density of $3 \mathrm{D}$ images in (e) (three areas per mouse, $n=4 \mathrm{mice} /$ group). Data are shown as mean $\pm \mathrm{SEM}$. $* * p<0.01$, $* * * p<0.001, * * * * p<0.0001$; two-way ANOVA with Tukey's multiple comparison (graphs in b, d) and two-tailed Student's $t$-test (graph f)

7, the Plxnd $1^{i E C K O}$ mice generally showed significantly decreased neuron numbers (Fig. 2i).

\section{Altered Structural and Functional Cerebrovascular Remodeling in ${ }^{\text {Plxnd1 iECKO }}$ Mice after Stroke}

To investigate whether reactivation of Sema3E/Plexin-D1 signaling following stroke leads to vascular alterations, we first monitored $\mathrm{CBF}$ in the ischemic region using laser 
a
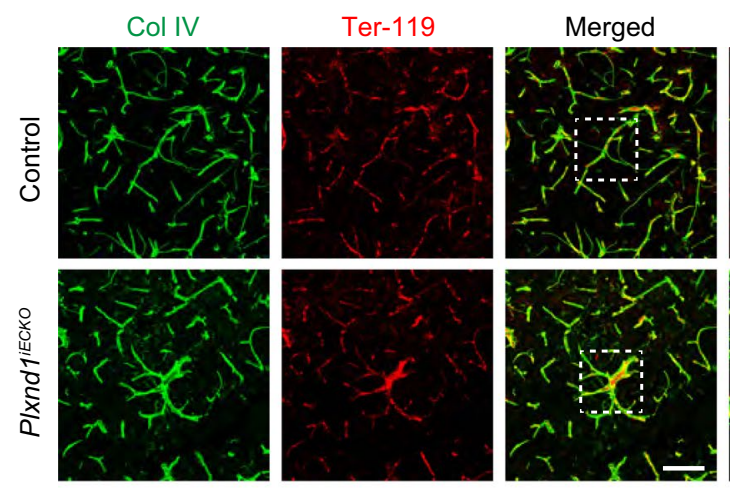

C

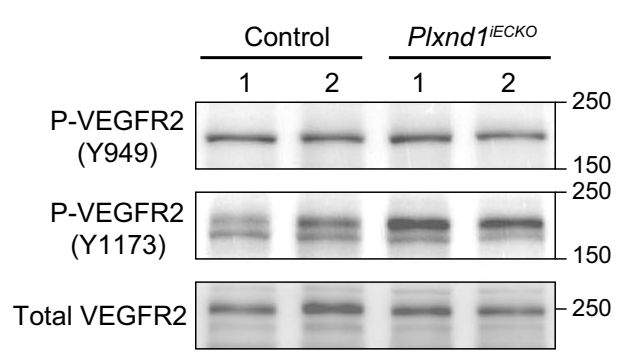

e

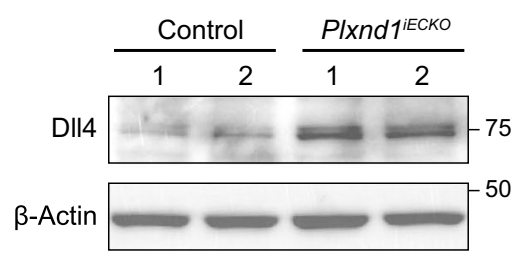

b
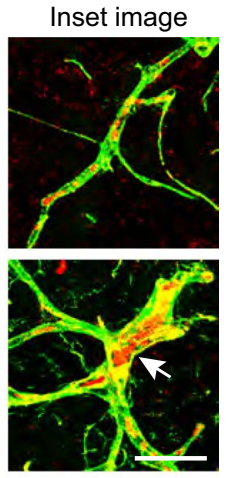

d
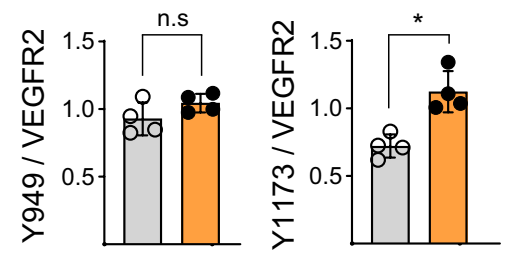

f

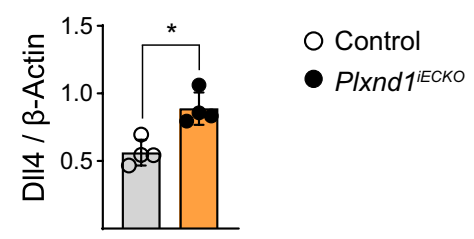

Fig. 4 Lack of Plxnd 1 in brain endothelial cells increases VEGF signaling leading to abnormal vascular morphology a Representative images of microvessel structure in the peri-infarct region at posttMCAo day 7 from control (Cre positive Plxnd $1^{f l o x /+}$, top panels) and Plxnd $l^{i E C K O}$ (bottom panels) mice. During recovery after stroke induction, the control mice showed typical capillary vessel structure (green, anti-Col IV) with single red blood cell inner lining (red, antiTer-119), whereas the Plxnd1 ${ }^{i E C K O}$ mice generated unusually thick vessels with accumulated RBCs (white arrow in bottom inset image). Boxed areas in merged panels are shown in high-resolution images (inset). Scale bars $=100 \mu \mathrm{m}$; high-resolution images $=50 \mu \mathrm{m}$. b

Doppler imaging (Fig. 3a). No significant difference was observed in CBF between control and Plxnd $I^{i E C K O}$ groups before (baseline) or after occlusion (tMCAo) onset. In contrast, at 7 days after reperfusion, CBF recovery was delayed by $\sim 20 \%$ in Plxndl $l^{i E C K O}$ mice when compared with control littermates (Fig. 3b).

In a rodent stroke model, active vessel sprouting begins 3 days after ischemic stroke, and noticeable angiogenesis is observed 1 week later $[14,46]$. Therefore, we analyzed structural changes in the peri-infarct vasculature up to 7 days post-tMCAo. Ischemia significantly decreased the
Quantification of abnormal vessels from (a) ( $n=5$ mice/group). Open and closed circles in black indicate control and Plxnd ${ }^{i E C K O}$ mice, respectively (graphs in b, d, f). c Phosphorylation level of Y949 and Y1173 sites including total VEGFR2 expression analyzed by western blot at post-tMCAo day 4. Representative blots show separate periinfarct samples from different animals per each genotype. d Quantification of phospho-tyrosine levels normalized by VEGFR2 expression in (c) ( $n=4$ mice/group). e Western blot images of Dll4 expression using the same samples in (c). f Quantification of Dll4 level normalized by $\beta$-actin expression ( $n=4$ mice/group). Data are shown as mean \pm SEM. ${ }^{*} p<0.05$, ${ }^{* *} p<0.01$; two-tailed Student's $t$-test

vessel number by day 3 in both heterozygous control and Plxnd $I^{i E C K O}$ mice; however, vessel number recovery was only measured in the control mice at post-tMCAo day 7 (Fig. 3c, d). Plxndl ${ }^{i E C K O}$ mice showed a significant decrease in vessel density at day 7 compared with control mice. Interestingly, we observed that Plxndl $I^{i E C K O}$ mice had increased overall vessel diameter and a few aberrantly thick vessels despite reduced vascular formation (Fig. 3c, d). These results suggested that Plexin-D1 expression in damaged vessels 3 days after tMCAo (Fig. 1d) is crucial for angiogenic sprouting and subsequent vascular network repair. 
For further in-depth analysis of the vasculature defect, we performed three-dimensional (3D) image analysis using the CUBIC method, a recently developed tissue-clearing technique [42] that allows visualization of the entire vascular network surrounding the infarct core (Fig. 3e). Overall, 3D vascular patterning was much less elaborate in the Plxnd $1^{i E C K O}$ mice; all parameters were significantly lower than those of the control mice (Fig. 3f). This emphasized that brain endothelial Plexin-D1 promotes vascular network formation following ischemic stroke.

\section{Aberrant VEGF Signaling Impairs Vascular Remodeling in ${ }^{\text {Plxnd1 } 1 \text { iECKO }}$ Mice after Stroke}

Plxnd $I^{\text {iECKO }}$ mice displayed malformed blood vessels with microaneurysm-like abnormal structures (Fig. 4a, b), suggesting a misregulation of endothelial cell proliferation and/ or migration during vascular remodeling. VEGF/VEGFR2 signaling is a master regulatory pathway for vascular development and function in health and disease [47]; therefore, we assumed that Sema3E-Plexin-D1 controlled VEGF signaling after stroke. First, we examined VEGF signaling activity by measuring phosphorylated VEGFR2 expression 4 days post-tMCAo, when angiogenic remodeling actively progresses. We focused on two major tyrosine phosphorylation sites, Y949 and Y1173, that are associated with endothelial cell proliferation and migration [48]. Surprisingly, no change in the expression of phosphos-Y949 was found in the Plxndl ${ }^{i E C K O}$ mouse brain. In contrast, phosphorylation at Y1173 was significantly increased when compared with the control mice (Fig. 4c, d). To further assess the increased VEGF signaling, we examined Dll4 expression, a well-characterized VEGF downstream target in endothelial cells [49]. Dll4 expression was upregulated in Plxnd $I^{i E C K O}$ mice (Fig. 4e, f), which demonstrated that the absence of Plexin-D1 causes aberrant activation of VEGF signaling in vessel remodeling post-ischemia.

\section{Endothelial-Specific PIxnd1 Deletion Leads to BBB Breakdown}

We examined whether the abnormal vessel formation observed in Plxndl ${ }^{i E C K O}$ mice during ischemic remodeling was accompanied by other functional impairments, such as BBB breakdown. To assess the integrity of growing vessels during remodeling, we assessed BBB breakdown via T1-Gd MRI in live animals. Signal intensities increased in Plxndl ${ }^{i E C K O}$ mice when compared with control littermates (Fig. 5a, b), indicating incomplete BBB recovery in the absence of Plexin-D1. Next, we intravenously administered a low molecular weight $10-\mathrm{kDa}$ fluorescent dextran tracer and monitored its distribution within the injured cortex until post-tMCAo day 7. Tracer extravasation was observed on day 3 , which continuously increased until day 7 in Plxnd I IECKO mice. In control mice, $10-\mathrm{kDa}$ dye leakage was barely detectable even 7 days post-tMCAo (Fig. 5c, d). In addition, we evaluated whether the impaired vessels in Plxndl knockout mice were permeable to high molecular weight proteins, such as immunoglobulin $\mathrm{G}$, and found a significant accumulation in the brain parenchyma (Fig. 5e, f).

To further investigate whether BBB disruption was caused by a structural change of the NVU, the expression of three major BBB junctional proteins was analyzed in the periinfarct region. The expression of junctional proteins, ZO-1, Claudin5, and Occludin, was downregulated in Plxnd $I^{i E C K O}$ mice when compared with sham or control mice (Fig. 6a, b). Despite their overall downregulation in knockout mice, ZO-1 and Claudin5 were detected on remodeling vessels albeit with an altered distribution (Fig. 6c, arrowheads). The coverage ratio of Occludin on vessels lacking Plexin-D1 was extremely low (Fig. 6c arrows, d). These results demonstrated that Plexin-D1 signaling is critical to establish proper brain vascular structures because it controls the expression and distribution of BBB constituents in growing vessels.

\section{Inhibition of VEGF Signaling during Vascular Remodeling Facilitates Functional Recovery in Plxnd 1 iECKO Mice}

Based on the above results, we speculated that reactivation of Plexin-D1 may control VEGF signaling in remodeling vessels, thereby preventing the detrimental effects of overactivated VEGF. To test this, we administered SU5416, a VEGFR2 inhibitor, during the vascular remodeling period after stroke (Fig. 7a) and analyzed VEGFR2 activation and its downstream target D114 expression. SU5416 injection dramatically reduced phosphor-Y1173 and D114 expression in Plxnd ${ }^{i E C K O}$ mice (Fig. 7b, c). These results provided additional evidence that signaling through VEGF2 activation in the remodeling vessel is closely modulated by the Plexin-D1 signaling pathway at the molecular level. Next, we monitored body weight and neurological score. As shown in Fig. 7d, the VEGFR2 inhibitor treatment prevented body weight loss and improved behavioral performance in Plxndl $I^{i E C K O}$ mice when compared with vehicle-treated Plxndl $I^{i E C K O}$ mice. This indicated that the functional recovery of Plxnd $1^{i E C K O}$ mice was inhibited by overactive VEGFR2 signaling. In Plxnd $I^{i E C K O}$ mice, VEGFR2 inhibition reduced infarction area (Fig. 7e, f) and BBB permeability (Fig. 7g, h). Moreover, SU5416 treatment reverted the abnormal vascular morphology in Plxnd ${ }^{i E C K O}$ mice (larger diameter and reduced vascular density) (Fig. 7g, i).

Finally, we tested whether the inactivation of VEGFR2 signaling within a specific time window was critical to stroke recovery. We administered SU5416 to wild-type mice before or 
a

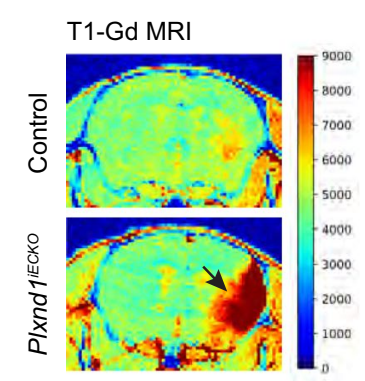

C

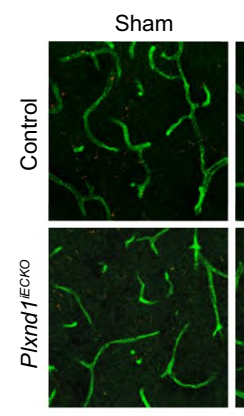

CD31/ $10 \mathrm{kDa}$ tracer

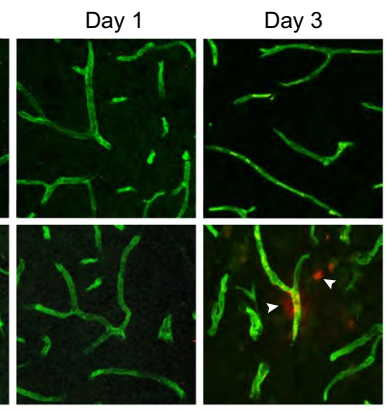

e

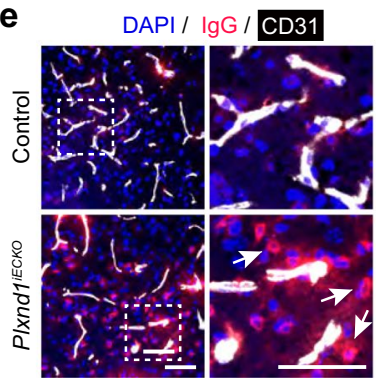

Day 7

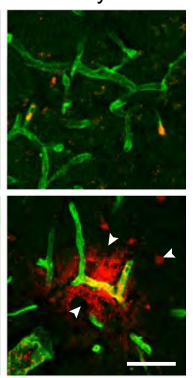

f b

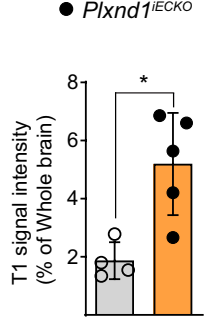

d

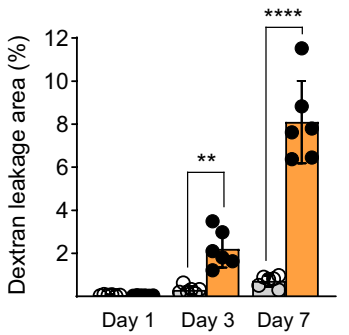

Fig. 5 BBB breakdown during ischemia-induced vessel remodeling in Plxnd1 knockout mice. a Representative T1-Gd MRI image. Intensity of signal change between control (Cre positive Plxnd1 $1^{\text {flox/++ }}$ ) and Plxndl ${ }^{\text {iECKO }}$ mouse brain parenchyma (jet image colormap). Black arrows indicate penetrable Gd-DOTA distribution in the PlxndI ${ }^{i E C K O}$ mice. b Quantitative analysis showing that Gd-DOTA enhancement was increased in the brain parenchyma of PlxndiECKO mice, indicating increased $\mathrm{BBB}$ permeability $(n=4 \mathrm{mice} / \mathrm{control}$ group, $n=5$ mice/Plxnd $1^{i E C K O}$ group). Open and closed circles in black indicate control and Plxndl ${ }^{i E C K O}$ mice, respectively (graphs in $\mathbf{b}$, d, f). c Confocal microscopy imaging of $10-\mathrm{kDa}$ dextran tracer (red) around CD31-positive capillaries (green) in control (top panels) and Plxndl ${ }^{i E C K O}$ (bottom panels) mice. Considerable tracer leakage into the brain parenchyma of Plxndl $I^{i E C K O}$ mice appeared as early as post-

after vascular remodeling began and analyzed the brain damage. The early inactivation of VEGF signaling caused severe brain damage, including increased edema and infarction area $(\sim 30 \%)$ even at day 4 post-tMCAO (Supplemental Fig. 5a-e). Moreover, late VEGF signaling inactivation during vascular recovery also caused brain damage; however, its severity was less mild ( 15\% infarction area, Supplemental Fig. 5f-j) than the early inactivation. These results indicated that VEGF signaling activation after ischemia is necessary for proper vascular remodeling, but its activation degree is differently regulated.

\section{Discussion}

This study demonstrated that the reactivation of Sema3EPlexin-D1 signaling following ischemic stroke is essential for reconstructing a healthy vasculature via the regulation of VEGF signaling during vascular remodeling. Therefore, enhancing Sema3E-Plexin-D1 signaling in angiogenic

tMCAo day 3, whereas permeability in the control mice was barely detectable. Arrowheads indicate accumulated tracer in perivascular tissues. Scale bar $=50 \mu \mathrm{m}$. d Quantification of vascular permeability ( $n=6$ mice/group). Tracer accumulated areas were analyzed as the relative percentage of sham control (Cre positive Plxnd $1^{\text {flox/+ }}$ without stroke induction) values. e Immunostaining of $\operatorname{IgG}$ (red) and CD31 (white) at post-tMCAo day 7. Dotted boxes in the left panels are shown in high-resolution images on the right panels. The white arrows indicate accumulated leak $\mathrm{IgG}$ in the brain parenchyma of Plxnd $I^{i E C K O}$ mice. Scale bar $=100 \mu \mathrm{m}$. f Quantification of IgGstained areas $(n=5 \mathrm{mice} /$ group $)$. Data are shown as mean $\pm \mathrm{SEM}$. $* p<0.05, * * p<0.01, * * * * p<0.0001$; two-tailed Student's $t$-test (graphs in $\mathbf{b}$ and $\mathbf{f}$ ) and two-way ANOVA with Tukey's multiple comparison (graph d)

vessels during the critical period of vascular repair could become a new target for stroke treatment.

The expression of Sema3E and Plexin-D1 in the nervous and vascular systems changes dramatically after birth (Supplemental Fig. 1) [34]. In the developing brain, Plexin-D1 was broadly detected across capillary endothelial cells. At birth, however, its expression in vessels was sharply downregulated, and it was barely evident in adult brain vasculature (Fig. 1 and Supplemental Fig. 1). Our previous findings have demonstrated that unlike in the developing brain or peripheral vasculature, in the developing mouse retina, Plxndl mRNA is expressed only in endothelial cells at the front of actively sprouting blood vessels from P3 to P5 and reappears in new sprouts to form deeper vascular layers at P6 [36, 37]. Those results, together with the results of the present study, strongly suggest that Plexin-D1 expression is practically absent in mature blood vessels. 
Fig. 6 BBB breakdown in Plxndl knockout mice is accompanied by reduced junctional protein expression and distribution a Representative western blot images of ZO-1, Claudin5, and Occludin in periinfarct regions on post-tMCAo day 7. b Quantification of three junctional protein expressions shown in (a). Each expression level is normalized to $\beta$-Actin ( $n=5$ mice/group). Open triangles, open circles, and closed circles in black indicate sham-operated, control, and Plxnd $1^{i E C K O}$ mice, respectively (graphs in b, d). c Representative images of ZO-1 (green) and CD31 (red); Claudin5 (green), and CD31 (red); and Occludin (green) and CD31 (red) staining of the peri-infarct region on post-tMCAo day 7. Merged results are shown in larger images. In the Plxndl $1^{\text {iECKO }}$ mice, some ZO-1 and Claudin-5 proteins are distributed on vessels (white arrowheads) whereas Occludin localization on vessels is barely detected (white arrows) compared with sham (Cre positive Plxnd $1^{f l o x /+}$ without stroke induction) or control mice. Scale bar in larger images $=50 \mu \mathrm{m}$. d Quantification showing ZO-1, Claudin5, and Occludin coverage in the peri-infarct region $(n=8$ mice/group). Data are shown as mean \pm SEM. $* * p<0.01$, $* * * p<0.001, * * * * p<0.0001$; one-way ANOVA with Tukey's multiple comparison

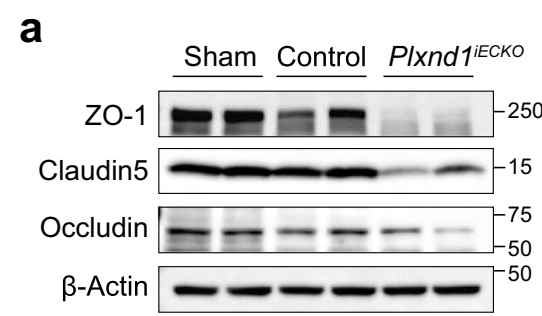

b

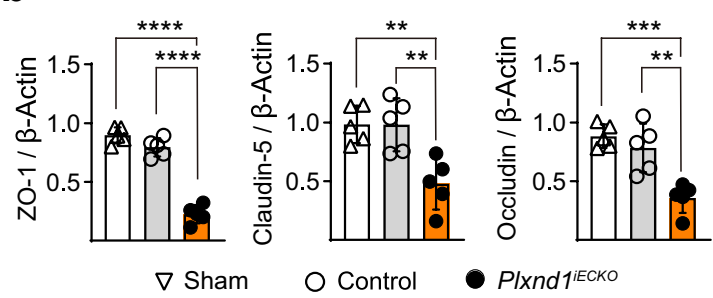

C
Sham
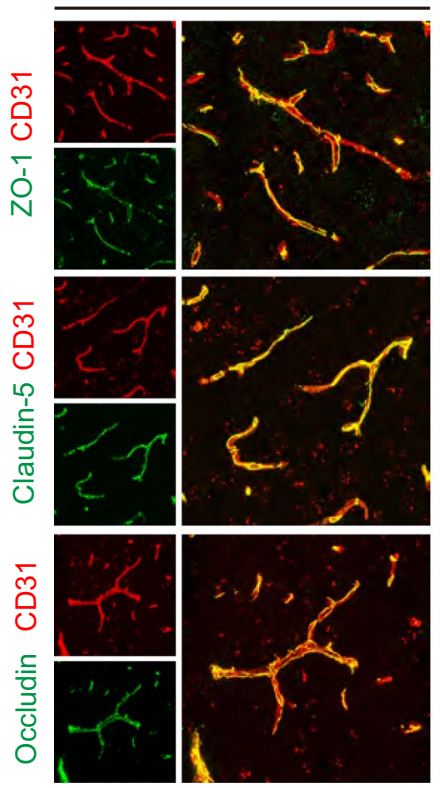

Control
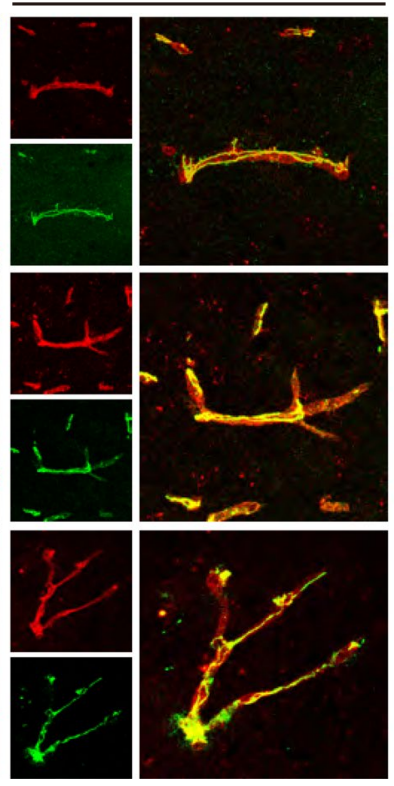

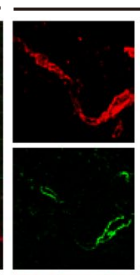

Plxnd1 1ECKO

d
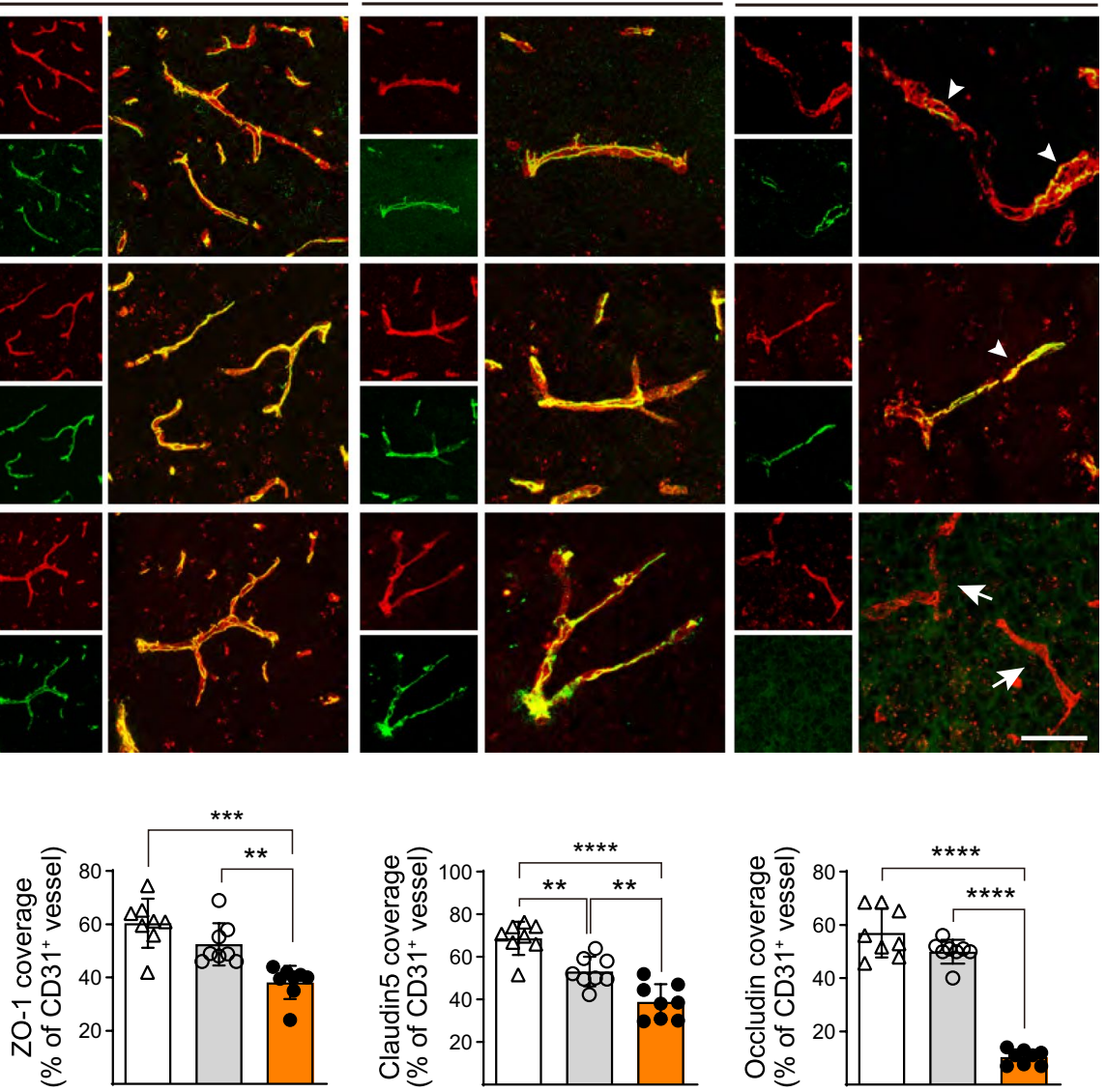

$\triangle$ Sham $\bigcirc$ Control Plxnd1 1ECKo
Interestingly, Plexin-D1 expression is regulated by VEGF signaling, a major hypoxia-inducible pathway, during retinal vascularization [36]. Furthermore, it is highly upregulated in the endothelial cells of extraretinal vessels in the ischemic retina [37]. Therefore, we speculated that Plexin-D1 expression is essential in critical situations, such as vascular remodeling induced by ischemia, where newly sprouting vessels require vascular guidance in response to VEGF signaling [50]. Previous reports that have used the same ischemic stroke model found that VEGF expression was remarkably elevated in the cortex within $3 \mathrm{~h}$ post-ischemia. In contrast, VEGFR2 expression was high on day 1 and remained high until post-ischemia day 21 [14]. Consistent with this, vascular Plexin-D1 was observed in our study at post-ischemic insult day 3 and reached most of the damaged area in similar ischemic conditions in this study (Fig. 1d, e). This supports our assumption that Plexin-D1 expression is under VEGF signaling control, even in the brain vasculature.

Sema3E expression is confined to specific neuronal cells in the brain from development to adulthood [34, 51]. Previous studies have documented differential Sema3E expression and function under various ischemic conditions. For example, Sema3E and Plexin-D1 are upregulated in ischemic mouse limbs via p53. Inhibition of Sema3E function leads to a significant increase in blood flow recovery [52], indicating that Sema3E/Plexin-D1 signaling activation adversely affects neovascularization. In a murine ischemic retinopathy model, Sema3E expression in ganglionic cells is 
a

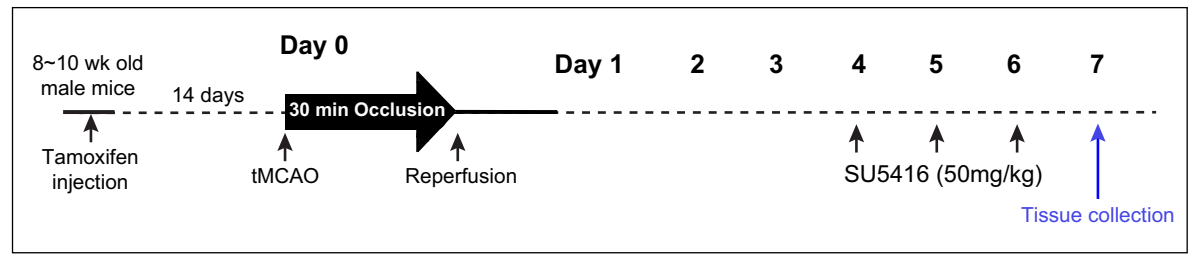

b

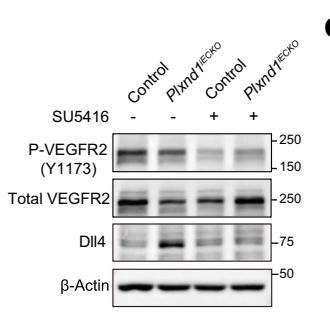

C

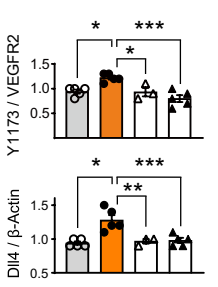

e

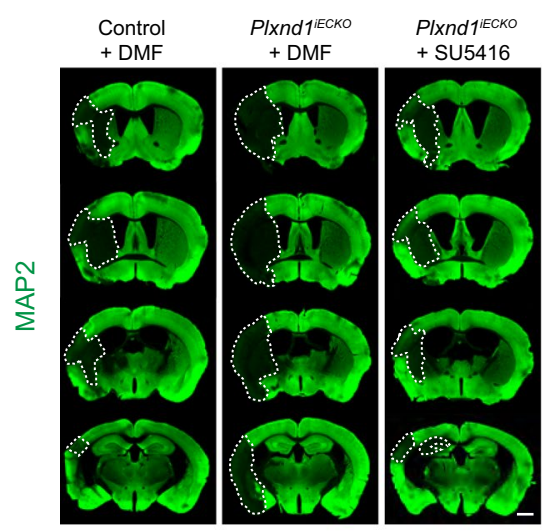

i

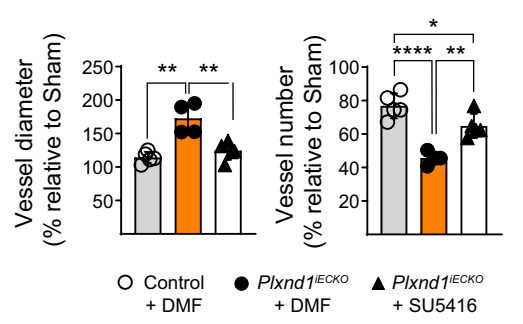

Fig. 7 Inhibition of VEGF signaling enhances functional recovery and vascular remodeling in Plxndl knockout mice a Schematic illustration of the experimental strategy for (b) to (i). b Representative western blots showing that injection of SU5416 rescued the aberrant expression of phosphorylated VEGF-R2 (Y1173) and Dl14 in the peri-infarct cortex of Plxnd1 ${ }^{i E C K O}$ mice on post-tMCAo day 7. c Quantification from (b). Each symbol marks the following (graphs in $\mathbf{c}, \mathbf{d}, \mathbf{f}, \mathbf{h}, \mathbf{i}$ ): dimethylformamide (DMF)-administered control (open circles) and Plxnd1 ${ }^{\text {EECKO }}$ mice (closed circles); VEGFR2 inhibitor, SU5416 administered-control (open triangles), and PlxndI ${ }^{i E C K O}$ (closed triangles) mice. d Body weight and neurological scores were monitored for 7 days after tMCAo ( $n=4-6$ mice/group). $* p<0.05$, ** $p<0.001, \quad * * * p<0.001$; control versus Plxndi ${ }^{i E C K O}+\mathrm{DMF}$ groups, $\quad \# p<0.05, \quad \# \# p<0.01 ; \quad$ Plxnd $1^{i E C K O}+$ DMF versus Plxnd1 ${ }^{i E C K O}+$ SU5416 groups. e Representative images of an infarction area. Brain sections were collected at post-tMCAO day 7, and the damaged brain area was analyzed by MAP2 immunostaining. Dotted d $\triangle$ Control+DMF $\triangle$ Control+SU5416

- Plxnd1 ${ }^{1 E C K O}+$ DMF $\Delta$ Plxnd1 ${ }^{1 E C K O}+$ SU5416

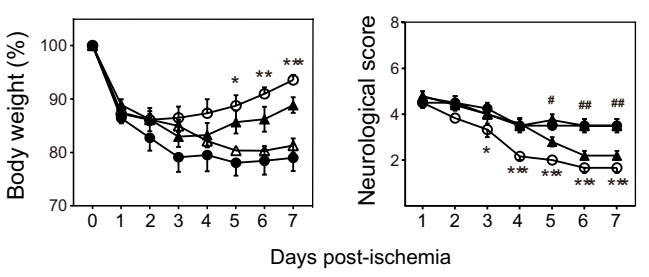

$\mathbf{f}$

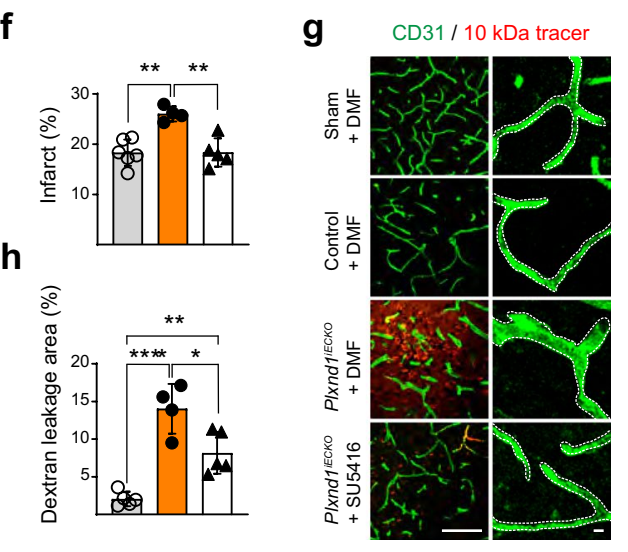

h

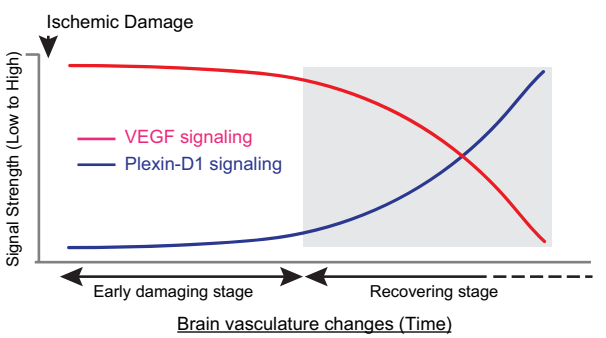

lines indicate the infarction region. Scale bar $=1 \mathrm{~mm}$. f Quantification of infraction area. $\mathbf{g}$ Representative images of vascular permeability assay (left panels) after $10-\mathrm{kDa}$ dextran tracer (red) injection. In Plxnd $I^{\text {iECKO }}$ mice, vehicle injection shows significant tracer leakage into the brain parenchyma, whereas SU5416 administration decreases tracer permeability. The dye leakage is barely detectable in the sham or control mice. Magnified images (right panels) show the CD31positive vascular structure (green). The dotted lines demarcate vessel shape. Scale bar $=100 \mu \mathrm{m}$. h Quantification of dye leakage. The leakage area analyzed the relative percentage to sham control (Cre positive Plxnd $1^{f l o x /+}$ without stroke induction) data. i Quantification of vessel diameter and number changes in $(\mathbf{g})(n=5 \mathrm{mice} / \mathrm{control}+\mathrm{DMF}$ and Plxndl $I^{i E C K O}+$ SU5416 groups, $n=4$ mice/Plxnd $I^{i E C K O}+\mathrm{DMF}$ group). Data are shown as mean \pm SEM. $* p<0.05$, $* * p<0.001$, $* * * * p<0.0001$; one-way ANOVA with Tukey's multiple comparison. $\mathbf{j}$ Schematic drawing describing the relative changes of VEGF and Plexin-D1 signaling in our stroke model 
relatively constant. In contrast, reduced expression of aqueous Sema3E is observed in patients with diabetic retinopathy when compared with nondiabetic controls [37, 53, 54]. This suggests that the regulatory activity of Sema3E varies by pathological condition and species. In the present study, Sema3E expression was rapidly and precisely induced in peri-infarct area neurons during the early phase after stroke (Fig. 1a-c). Therefore, further study should examine how Sema3E expression is elevated in such a short period in the hypoxic brain.

Multiple studies have demonstrated that axon guidance molecules are associated with modulating vascular patterning under disease conditions and during development via similar signaling pathways $[12,55]$. These guidance cues may be beneficial or detrimental during remodeling in the ischemic brain [56-60]. Sema3A has been studied during vascular recovery following ischemic damage. Stroke induction increases Sema3A in the cortex for up to 2 weeks and is detrimental to the damaged brain [61]. Additionally, Sema3A injection into the cerebral cortex increases vascular permeability, and the ablation of Sema3A expression reduces cerebrovascular permeability and brain damage in the tMCAO model [62]. Similarly, Sema3E may function as a remodeling modulator. In an oxygen-induced retinopathy model, enhanced Plexin-D1 or intravitreal Sema3E injection prevents abnormal vessel projections and leads to normal vascular remodeling [37]. This suggests a beneficial mechanism of Sema3E-PlexinD1. In an ischemia-induced aged rat brain model, Zhou et al. have reported that the inhibition of Sema3E-PlexinD1 signaling via a lentiviral shRNA knockdown system recovers the vascular network and improves physiological functions, such as cerebral perfusion and BBB integrity, thereby reducing brain damage [63]. However, the results from our current study, using genetically modified mouse models, demonstrated that a lack of Sema3E-Plexin-D1 signals in the ischemic brain leads to significant tissue damage and delayed recovery (Fig. 2). This strongly suggests a beneficial role for Sema3E-Plexin-D1 during stroke-induced functional recovery.

The underlying mechanism of Sema3E/Plexin-D1 signaling involvement in functional brain recovery after ischemic stroke is unclear. Our 3D-vascular network analysis and blood flow recovery experiment indicated that the greatest damage in the brains of Plxndl knockout mice was due to aberrant and decreased vasculature (Fig. 3, 4). Previously, the absence of this signaling pathway was thought to unlock the normal limits on vessel growth, thereby increasing the size and branching of vascular networks. This has been observed in mouse peripheral and avian periocular vasculature; vessels devoid of Sema3E or Plexin-D1 grow densely across their boundaries [13, 64]. However, this mechanism is not observed in the retinal vasculature, where Sema3e or Plxndl knockout results in sparse and underdeveloped network formation. This indicates that the diverse regulatory behaviors of Sema3E-Plexin-D1 signaling are dependent upon location and tissue. In our study, Plxndl knockout mice had reduced vasculature during remodeling as opposed to the overgrowth observed in developing vasculature. Therefore, they recapitulated the retinal vasculature devoid of Sema3e or Plxndl knockouts [36, 37].

In addition, Plxndl knockout mice showed aberrantly thick remodeling vessels, which may have been caused by abnormal endothelial cell proliferation and migration (Fig. 4a, b). We analyzed phospho-VEGFR2 expression at Y1173, which is the site responsible for regulating endothelial cell proliferation and migration [48]. We found increased phosphorylation in the Plxndl knockout mice (Fig. 4c, d). In addition, we examined Dll4 expression, which is associated with normal sprouting and branching as an intercellular VEGF signaling mediator [36]. Its expression was significantly increased in the Plxndl knockout mice (Fig. 4e, f). These results are consistent with the previous finding that Sema3E-Plexin-D1 negatively controls VEGF signaling in the retina vasculature [36]. Considering the numerous phosphorylation sites on VEGFR2, it will be interesting to explore how the Y1173 site is under the control of Plexin-D1 signaling. Recently, Fukushima et al. [38]. have reported that Sema3E treatment results in VEGFR2 phosphorylation at Y1214, but not Y1175 (comparable to mouse Y1173), which induces reverse migration in cultured human endothelial cells. Moreover, this event occurs via RhoJ-mediated Plexin-D1 and VEGFR2 association. Therefore, it will be interesting to study whether this mechanism is recapitulated during cerebrovascular remodeling in our ischemic model.

During stroke, the VEGF signaling pathway shows both positive and negative effects in the ischemic brain according to their activation timing and dose across the initial-to-late chronic conditions [29]. Given the rapid VEGF and VEGFR2 expression after ischemic induction [14], VEGF signaling is necessary in the acute phase after stroke onset, probably acting for neuroprotection and angiogenic potentiation despite the high risk of a leaky BBB. Later, the newly sprouting vessels need to optimize the overactivated VEGF signaling to maintain proper angiogenic strength and neuroprotective roles via Sema3E-Plexin-D1. Supporting this assumption, we showed that VEGF signaling inhibition during the vascular remodeling phase promoted functional recovery accompanied by relatively normal vasculature in the Plxndl knockout mice (Fig. 7). By contrast, VEGF signaling inhibition in the wild-type mice after stroke aggravated brain damage with a slight difference according to the inactivation time (Supplemental Fig. 5). In 
conclusion, VEGF signaling is immediately activated and prepares angiogenic sprouting during the early stages after ischemic damage, but its continuous overactivation during the recovery stage can lead to vascular injuries. To prevent the harmful effects of VEGF signaling, the reactivation of Sema3E-Plexin-D1 signaling is necessary as the damaged vasculature enters the remodeling stage (Fig. 7j). Plexin-D1 deficiency may cause excessively active VEGF signaling and induce functional and structural vascular malformations.

In this study, the disruption of Sema3E/Plexin-D1 signaling caused severe BBB breakdown. This was signified by increased tracer permeability and fewer junctional proteins (Figs. 5 and 6). BBB breakdown in response to ischemia occurs in two sequential steps: upregulation of transcytosis within hours, followed by paracellular pathway defects through the remodeling of tight junction proteins in the next 2-3 days [65]. Considering the timing of Plexin-D1 reemergence, we speculate that Sema3E-Plexin-D1 signaling is more relevant to BBB permeability through the paracellular pathway. Another mode of BBB disruption comes from the interaction impairment between endothelial cells and pericytes in the brain. During development, both cells have a reciprocal interaction through a specific signaling pathway. Endothelial cells secrete PDGF-BB to recruit pericytes expressing PDGFR- $\beta$. Conversely, pericytes secrete angiopoietin 1 (Ang1) to activate endothelial cells via Tie2 receptors [66]. Furthermore, pericytes cover most brain capillaries and continue producing matrix proteins in the basement membrane alongside endothelial cells. This promotes a stable BBB structure and impermeability [67]. Intriguingly, pericyte-derived Ang1 induces Occludin expression in brain capillary endothelial cells [66]. In this study, we found that Occludin distribution was exclusively reduced in the absence of Sema3E/Plexin-D1 signaling (Fig. 6c), which suggested a potential relationship between BBB disruption and abnormal pericytes in the Plxndl knockout mice. Collectively, future studies should explore the involvement of Sema3E/Plexin-D1 signaling in the pericyte coverage process during vascular remodeling after stroke.

Here, we showed the antagonistic mechanisms of Sema3E-Plexin-D1 signaling as a beneficial mediator on VEGF-induced angiogenic sprouting. Further attention should focus on how these signaling pathways are precisely interconnected during vascular remodeling after stroke. Finally, further examination of the specific downstream targets of Sema3E-Plexin-D1 signaling in brain endothelial cells should be conducted to decipher the molecular mechanisms of intercellular communication. This may uncover novel therapeutic strategies for ameliorating the unfavorable outcomes in brain vascular diseases, such as stroke.

\section{Conclusion}

In summary, we show that Sema3E-Plexin-D1 signaling is necessary for the development of functional brain vasculature during vascular remodeling after an ischemic insult. We found that after ischemia, neurons in the peri-infarct region upregulate Sema3E, and vessels close to the damaged region subsequently express Plexin-D1. Furthermore, loss of Plexin-D1 function exacerbated brain damage and abnormal behavioral performance, accompanied by lower vessel density in the periinfarct region, similar to previous observations in a retinopathy model [37]. Newly generated vessels lacking Plexin-D1 also yielded abnormal BBB function and structure. Suppression of overactive VEGF signaling in the Plxndl knockout during vascular remodeling periods rescued vascular defects. These data support a beneficial role of Sema3E-Plexin-D1 signaling in vascular repair regulation following ischemic stroke.

Abbreviations AP: Alkaline phosphatase; BBB: Blood-brain barrier; CBF: Cerebral blood flow; CCA: Common carotid artery; ICA: Internal carotid artery; ISH: In situ hybridization; MCAo: Middle cerebral artery occlusion; MRI: Magnetic resonance imaging; NVU: Neurovascular unit; PFA: Paraformaldehyde; SEM: Standard error of the mean; VEGF: Vascular endothelial growth factor

Supplementary Information The online version contains supplementary material available at https://doi.org/10.1007/s12975-021-00914-4.

Acknowledgements We thank Drs. Ayal Ben-Zvi, Masha Gelfand, and Baptiste Lacoste for reading the manuscript and providing critical feedback; Dr. Chenghua Gu for providing Plxnd1-flox mice; the Advanced Neural Imaging Center in KBRI for light sheet and confocal microscopy image analysis; and Dr. Hoe-Su Jung of the Laboratory Animal Center at Daegu-Gyeongbuk Medical Innovation Foundation for MRI analysis.

Author Contribution WO conceived and designed the project. WO and RY conducted experiments and acquired data. RY, JJ, YL, and NK analyzed imaging data and quantification. BZ provided the mfsd2aCreERT2 mouse model. SP performed data interpretation. WO and RY wrote the manuscript with significant input from SP.

Funding This research was supported by the KBRI basic research program of the Korea Brain Research Institute funded by the Ministry of Science and ICT (KBRI 20-BR-01-06) and the Bio \& Medical Technology Development Program of the National Research Foundation (NRF) \& funded by the Korean government (MSIT) (NRF-2020M3E5D9079766).

Data availability All data generated and analyzed for this study are included in this published article and its supplementary additional files.

\section{Declarations}

Ethics approval All protocols for animal experiments were approved by the Institutional Animal Care and Use Committee of Korea Brain Research Institute (IACUC-18-00008). All experiments were performed according to the National Institutes of Health Guide for the Care and Use of Laboratory Animals and ARRIVE guidelines. 
Consent for publication Not applicable.

Conflict of Interests The authors declare no competing interests.

Open Access This article is licensed under a Creative Commons Attribution 4.0 International License, which permits use, sharing, adaptation, distribution and reproduction in any medium or format, as long as you give appropriate credit to the original author(s) and the source, provide a link to the Creative Commons licence, and indicate if changes were made. The images or other third party material in this article are included in the article's Creative Commons licence, unless indicated otherwise in a credit line to the material. If material is not included in the article's Creative Commons licence and your intended use is not permitted by statutory regulation or exceeds the permitted use, you will need to obtain permission directly from the copyright holder. To view a copy of this licence, visit http://creativecommons.org/licenses/by/4.0/.

\section{References}

1. Benjamin EJ, Virani SS, Callaway CW, Chamberlain AM, Chang AR, Cheng S, et al. Heart disease and stroke statistics-2018 update: a report from the American Heart Association. Circulation. 2018;137(12):e67.

2. Hu X, De Silva TM, Chen J, Faraci FM. Cerebral vascular disease and neurovascular injury in ischemic stroke. Circ Res. 2017;120(3):449-71.

3. Freitas-Andrade M, Raman-Nair J, Lacoste B. Structural and functional remodeling of the brain vasculature following stroke. Front Physiol. 2020;11:948.

4. Hatakeyama M, Ninomiya I, Kanazawa M. Angiogenesis and neuronal remodeling after ischemic stroke. Neural Regen Res. 2020;15(1):16

5. Arai K, Jin G, Navaratna D, Lo EH. Brain angiogenesis in developmental and pathological processes: neurovascular injury and angiogenic recovery after stroke. FEBS. 2009;276(17):4644-52.

6. Del Zoppo G. The neurovascular unit in the setting of stroke. J Intern Med. 2010;267(2):156-71.

7. Murphy TH, Corbett D. Plasticity during stroke recovery: from synapse to behaviour. Nat Rev Neurosci. 2009;10(12):861-72.

8. Gravanis I, Tsirka SE. Tissue-type plasminogen activator as a therapeutic target in stroke. Expert Opin Ther Targets. 2008;12(2):159-70.

9. Amani H, Habibey R, Shokri F, Hajmiresmail SJ, Akhavan O, Mashaghi A, et al. Selenium nanoparticles for targeted stroke therapy through modulation of inflammatory and metabolic signaling. Sci Rep. 2019;9(1):1-15.

10. Carmeliet $P$, Tessier-Lavigne $\mathrm{M}$. Common mechanisms of nerve and blood vessel wiring. Nature. 2005;436(7048):193.

11. Hawkins BT, Davis TP. The blood-brain barrier/neurovascular unit in health and disease. Pharmacol Rev. 2005;57(2):173-85.

12. Adams RH, Eichmann A. Axon guidance molecules in vascular patterning. Cold Spring Harbor perspect Biol. 2010;2(5):a001875.

13. Gu C, Yoshida Y, Livet J, Reimert DV, Mann F, Merte J, et al. Semaphorin 3E and plexin-D1 control vascular pattern independently of neuropilins. Science. 2005;307(5707):265-8.

14. Hayashi T, Saito A, Okuno S, Ferrand-Drake M, Dodd RL, Chan PH. Damage to the endoplasmic reticulum and activation of apoptotic machinery by oxidative stress in ischemic neurons. J Cereb Blood Flow Metab. 2005;25(1):41-53.

15. Beck H, Plate KH. Angiogenesis after cerebral ischemia. Acta neuropathol. 2009;117(5):481-96.
16. Liu J, Wang Y, Akamatsu Y, Lee CC, Stetler RA, Lawton MT, et al. Vascular remodeling after ischemic stroke: mechanisms and therapeutic potentials. Prog Neurobiol. 2014;115:138-56.

17. Kanazawa M, Takahashi T, Ishikawa M, Onodera O, Shimohata T, del Zoppo GJ. Angiogenesis in the ischemic core: A potential treatment target? J Cereb Blood Flow Metab. 2019;39:753-69.

18. Phng L-K, Gerhardt H. Angiogenesis: a team effort coordinated by notch. Dev Cell. 2009;16(2):196-208.

19. Eichmann A, Simons M. VEGF signaling inside vascular endothelial cells and beyond. Curr Opin Cell Biol. 2012;24(2):188-93.

20. Karaman S, Leppänen V-M, Alitalo K. Vascular endothelial growth factor signaling in development and disease. Development. 2018;145.

21. Hayashi T, Abe K, Suzuki H, Itoyama Y. Rapid induction of vascular endothelial growth factor gene expression after transient middle cerebral artery occlusion in rats. Stroke. 1997;28(10):2039-44.

22. Lennmyr F, Ata KA, Funa K, Olsson Y, Terént A. Expression of vascular endothelial growth factor (VEGF) and its receptors (Flt-1 and Flk-1) following permanent and transient occlusion of the middle cerebral artery in the rat. J Neuropathol Exp Neurol. 1998;57(9):874-82.

23. Plate KH, Beck H, Danner S, Allegrini PR, Wiessner C. Cell type specific upregulation of vascular endothelial growth factor in an MCA-occlusion model of cerebral infarct. J Neuropathol Exp Neurol. 1999;58(6):654-66.

24. Sun Y, Jin K, Xie L, Childs J, Mao XO, Logvinova A, et al. VEGFinduced neuroprotection, neurogenesis, and angiogenesis after focal cerebral ischemia. J Clin Investig. 2003;111(12):1843-51.

25. Hayashi T, Abe K, Itoyama Y. Reduction of ischemic damage by application of vascular endothelial growth factor in rat brain after transient ischemia. J Cereb Blood Flow Metab. 1998;18(8):887-95.

26. Kirby ED, Kuwahara AA, Messer RL, Wyss-Coray T. Adult hippocampal neural stem and progenitor cells regulate the neurogenic niche by secreting VEGF. Proc Natl Acad Sci. 2015;112(13):4128-33.

27. Li W-L, Fraser JL, Shan PY, Zhu J, Jiang Y-J, Wei L. The role of VEGF/VEGFR2 signaling in peripheral stimulation-induced cerebral neurovascular regeneration after ischemic stroke in mice. Exp Brain Res. 2011;214(4):503.

28. Wang Y, Kilic E, Kilic Ü, Weber B, Bassetti CL, Marti HH, et al. VEGF overexpression induces post-ischaemic neuroprotection, but facilitates haemodynamic steal phenomena. Brain. 2005;128(1):52-63.

29. Geiseler SJ, Morland C. The Janus face of VEGF in stroke. Int J Mol Sci. 2018;19(5):1362.

30. Reeson P, Tennant KA, Gerrow K, Wang J, Novak SW, Thompson K, et al. Delayed inhibition of VEGF signaling after stroke attenuates blood-brain barrier breakdown and improves functional recovery in a comorbidity-dependent manner. J Neurosci. 2015;35(13):5128-43.

31. Lange C, Storkebaum E, De Almodóvar CR, Dewerchin M, Carmeliet P. Vascular endothelial growth factor: a neurovascular target in neurological diseases. Nat Rev Neurol. 2016;12(8):439.

32. Ding Q, Liao SJ, Yu J. Axon guidance factor netrin-1 and its receptors regulate angiogenesis after cerebral ischemia. Neurosci Bull. 2014;30(4):683-91. https://doi.org/10.1007/s12264-013-1441-9.

33. Oh WJ, Gu C. The role and mechanism-of-action of Sema3E and Plexin-D1 in vascular and neural development. Semin Cell Dev Biol. 2013;24(3):156-62. https://doi.org/10.1016/j.semcdb.2012. 12.001 .

34. Ding JB, Oh W-J, Sabatini BL, Gu C. Semaphorin 3E-PlexinD1 signaling controls pathway-specific synapse formation in the striatum. Nat Neurosci. 2012;15(2):215.

35. Chauvet S, Cohen S, Yoshida Y, Fekrane L, Livet J, Gayet O, et al. Gating of Sema3E/PlexinD1 signaling by neuropilin-1 switches 
axonal repulsion to attraction during brain development. Neuron. 2007;56(5):807-22.

36. Kim J, Oh WJ, Gaiano N, Yoshida Y, Gu C. Semaphorin 3E-Plexin-D1 signaling regulates VEGF function in developmental angiogenesis via a feedback mechanism. Genes Dev. 2011;25(13):1399-411. https://doi.org/10.1101/gad.2042011.

37. Fukushima Y, Okada M, Kataoka H, Hirashima M, Yoshida Y, Mann F, et al. Sema3E-PlexinD1 signaling selectively suppresses disoriented angiogenesis in ischemic retinopathy in mice. J Clin Invest. 2011;121(5):1974-85. https://doi.org/10.1172/JCI44900.

38. Fukushima Y, Nishiyama K, Kataoka H, Fruttiger M, Fukuhara S, Nishida $\mathrm{K}$, et al. RhoJ integrates attractive and repulsive cues in directional migration of endothelial cells. The EMBO J. 2020;39(12):e102930.

39. Pu W, Zhang H, Huang X, Tian X, He L, Wang Y, et al. Mfsd2a+ hepatocytes repopulate the liver during injury and regeneration. Nat Commun. 2016;7:13369.

40. Koizumi J. Experimental studies of ischemic brain edema. 1. A new experimental model of cerebral embolism in rats in which recirculation can be introduced in the ischemic area. Jpn $\mathrm{J}$ stroke. 1986;8(1):8

41. Xia C-F, Smith RS Jr, Shen B, Yang Z-R, Borlongan CV, Chao $\mathrm{L}$, et al. Postischemic brain injury is exacerbated in mice lacking the kinin B2 receptor. Hypertension. 2006;47(4):752-61.

42. Susaki EA, Tainaka K, Perrin D, Yukinaga H, Kuno A, Ueda HR. Advanced CUBIC protocols for whole-brain and whole-body clearing and imaging. Nat Protoc. 2015;10(11):1709.

43. Van Der Zwaag B, Hellemons AJ, Leenders WP, Burbach JPH, Brunner HG, Padberg GW, et al. PLEXIN-D1, a novel plexin family member, is expressed in vascular endothelium and the central nervous system during mouse embryogenesis. Dev Dyn. 2002;225(3):336-43.

44. Oh W-J, Gu C. Establishment of neurovascular congruency in the mouse whisker system by an independent patterning mechanism. Neuron. 2013;80(2):458-69.

45. Gitler AD, Lu MM, Epstein JA. PlexinD1 and semaphorin signaling are required in endothelial cells for cardiovascular development. Dev Cell. 2004;7(1):107-16.

46. Krupinski J, Stroemer P, Slevin M, Marti E, Kumar P, Rubio F. Three-dimensional structure and survival of newly formed blood vessels after focal cerebral ischemia. NeuroReport. 2003;14(8):1171-6.

47. Simons M, Gordon E, Claesson-Welsh L. Mechanisms and regulation of endothelial VEGF receptor signalling. Nat Rev Mol Cell Biol. 2016;17(10):611.

48. Koch S, Claesson-Welsh L. Signal transduction by vascular endothelial growth factor receptors. Cold Spring Harb Perspect Med. 2012;2(7):a006502.

49. Hellström M, Phng L-K, Hofmann JJ, Wallgard E, Coultas L, Lindblom P, et al. D114 signalling through Notch1 regulates formation of tip cells during angiogenesis. Nature. 2007;445(7129):776-80.

50. Marti HJ, Bernaudin M, Bellail A, Schoch H, Euler M, Petit E, et al. Hypoxia-induced vascular endothelial growth factor expression precedes neovascularization after cerebral ischemia. Am J Pathol. 2000;156(3):965-76.

51. Chauvet S, Mann F. The declaration of independence of the neurovascular intimacy. Neuron. 2013;80(2):262-5.

52. Moriya J, Minamino T, Tateno K, Okada S, Uemura A, Shimizu I, et al. Inhibition of semaphorin as a novel strategy for therapeutic angiogenesis. Circ Res. 2010;106(2):391-8. https://doi.org/10. 1161/CIRCRESAHA.109.210815.
53. Sun Y, Liu C-H, Wang Z, Meng SS, Burnim SB, SanGiovanni JP, et al. ROR $\alpha$ modulates semaphorin $3 \mathrm{E}$ transcription and neurovascular interaction in pathological retinal angiogenesis. FASEB J. 2017;31(10):4492-502.

54. Kwon SH, Shin JP, Kim IT, Park DH. Aqueous levels of angiopoietin-like 4 and semaphorin $3 \mathrm{E}$ correlate with nonperfusion area and macular volume in diabetic retinopathy. Ophthalmology. 2015;122(5):968-75.

55. Gelfand MV, Hong S, Gu C. Guidance from above: common cues direct distinct signaling outcomes in vascular and neural patterning. Trends Cell Biol. 2009;19(3):99-110.

56. Ghori A, Freimann FB, Nieminen-Kelhä M, Kremenetskaia I, Gertz K, Endres M, et al. EphrinB2 activation enhances vascular repair mechanisms and reduces brain swelling after mild cerebral ischemia. Arterioscler Thromb Vasc Biol. 2017;37(5):867-78.

57. Thundyil J, Manzanero S, Pavlovski D, Cully TR, Lok K-Z, Widiapradja A, et al. Evidence that the EphA2 receptor exacerbates ischemic brain injury. PLoS ONE. 2013;8(1):e53528.

58. Zhou Y-F, Li Y-N, Jin H-J, Wu J-H, He Q-W, Wang X-X, et al. Sema4D/PlexinB1 inhibition ameliorates blood-brain barrier damage and improves outcome after stroke in rats. FASEB J. 2018;32(4):2181-96.

59. Zheng M, Chen R, Chen H, Zhang Y, Chen J, Lin P, et al. Netrin-1 promotes synaptic formation and axonal regeneration via JNK1/cJun pathway after the middle cerebral artery occlusion. Front Cell Neurosci. 2018;12:13.

60. Rust R, Grönnert L, Weber RZ, Mulders G, Schwab ME. Refueling the ischemic CNS: guidance molecules for vascular repair. Trends in neurosciences. 2019.

61. Pekcec A, Yigitkanli K, Jung JE, Pallast S, Xing C, Antipenko A, et al. Following experimental stroke, the recovering brain is vulnerable to lipoxygenase-dependent semaphorin signaling. FASEB J. 2013;27(2):437-45.

62. Hou ST, Nilchi L, Li X, Gangaraju S, Jiang SX, Aylsworth A, et al. Semaphorin3A elevates vascular permeability and contributes to cerebral ischemia-induced brain damage. Sci Rep. 2015;5:7890.

63. Zhou Y-F, Li P-C, Wu J-H, Haslam JA, Mao L, Xia Y-P, et al. Sema3E/PlexinD1 inhibition is a therapeutic strategy for improving cerebral perfusion and restoring functional loss after stroke in aged rats. Neurobiol Aging. 2018;70:102-16.

64. Kwiatkowski SC, Ojeda AF, Lwigale PY. PlexinD1 is required for proper patterning of the periocular vascular network and for the establishment of corneal avascularity during avian ocular development. Dev Biol. 2016;411(1):128-39.

65. Knowland D, Arac A, Sekiguchi KJ, Hsu M, Lutz SE, Perrino J, et al. Stepwise recruitment of transcellular and paracellular pathways underlies blood-brain barrier breakdown in stroke. Neuron. 2014;82(3):603-17.

66. Hori S, Ohtsuki S, Hosoya Ki, Nakashima E, Terasaki T. A pericyte-derived angiopoietin-1 multimeric complex induces occludin gene expression in brain capillary endothelial cells through Tie-2 activation in vitro. J Neurochem. 2004;89(2):503-13.

67. Zhao Z, Nelson AR, Betsholtz C, Zlokovic BV. Establishment and dysfunction of the blood-brain barrier. Cell. 2015;163(5):1064-78.

Publisher's Note Springer Nature remains neutral with regard to jurisdictional claims in published maps and institutional affiliations. 